\title{
The mirror effect and the spacing effect
}

\author{
BENNET MURDOCK \\ University of Toronto, Toronto, Ontario, Canada
}

\begin{abstract}
In the mirror effect, there are fewer false negatives (misses) and false positives (false alarms) for rare (low-frequency) words than for common (high-frequency) words. In the spacing effect, recognition accuracy is positively related to the interval (spacing or lag) between two presentations of an item. These effects are related in that they are both manifestations of a leapfrog effect (a weaker item jumps over a stronger item). They seem to be puzzles for traditional strength theory and at least some current global-matching models. A computational strength-based model (EICL) is proposed that incorporates excitation, inhibition, and a closed-loop learning algorithm. The model consists of three nonlinear coupled stochastic difference equations, one each for excitation $(x)$, inhibition $(y)$, and context $(z)$. Strength is the algebraic sum (i.e., $s=x-y+z$ ). These equations are used to form a toy lexicon that serves as a basis for the experimental manipulations. The model can simulate the mirror effect forcedchoice inequalities and the spacing effect for single-item recognition, all parameters are random variables, and the same parameter values are used for both the mirror and the spacing effects. No parameter values varied with the independent variables (word frequency for the mirror effect, lag for the spacing effect), so the model, not the parameters, is doing the work.
\end{abstract}

The mirror effect and the spacing effect are two of the more puzzling phenomena in recognition memory. The mirror effect refers to the fact that low-frequency words have both a lower false alarm rate than do high-frequency words and a higher hit rate than do high-frequency words (Glanzer \& Adams, 1985). This would result if the four familiarity distributions were positioned as in Figure 1, where the low-frequency (or A) distributions flank the high-frequency (or B) distributions in a symmetric fashion. The mirror effect has also been found with other variables than word frequency, and there may be two kinds of mirror effects (Stretch \& Wixted, 1998), but I will restrict the analysis to the word frequency variable.

The spacing effect refers to the fact that, with two presentations of an item, the greater the spacing interval between the two presentations, the better the subsequent retention of the item. In the episodic memory area, this was first discovered in a study of the short-term retention of paired associates (Peterson, Wampler, Kirkpatrick, \& Saltzman, 1963) and was dubbed the Peterson paradox (Murdock, 1967), but it also occurs for recognition memory. Recognition response times for the second presentation (P2) increased with spacing between the first (P1)

This work was supported by Grant APA146 from the Natural Science and Engineering Research Council of Canada. I appreciate Dave Smith's help; he independently programmed up the forced-choice inequalities so we could get converging results from two semi-independent versions of the same routines. Also, thanks to Murray Glanzer, Bill Hockley, Mike Kahana, Kisok Kim, Roger Ratcliff, Sverker Sikström, and two referees for helpful correspondence on some of the issues in the paper. Correspondence concerning this article should be addressed to B. Murdock, Department of Psychology, University of Toronto, Toronto, ON, M5S 3G3 Canada (e-mail: murdock@ psych.utoronto.ca). and the second presentations (indicating forgetting), but response times for the third presentation or test (P3) decreased with spacing (Figure 2; Hintzman, 1969). This result nicely indicates the two aspects of the Peterson paradox: the greater forgetting of the first presentation, as shown by longer latencies for the P2 results, and an enhanced retention following the repetition, as shown by the shorter latencies for the $\mathrm{P} 3$ results.

\section{Leapfrog Effect}

These two effects (the mirror effect and the spacing effect) can be subsumed under what may be called a leapfrog effect. For the mirror effect, relative to high-frequency items, low-frequency items go from weaker to stronger after a single presentation. For the spacing effect, the weaker an item is after its first presentation, due to the longer retention interval, the stronger it is after its second presentation. Thus, for the mirror effect, after a single presentation the weaker low-frequency new item is stronger than an old high-frequency item, and for the spacing effect, the weaker (more forgetting of) an item is after its first presentation, the stronger (better learning) it is after its second presentation.

These effects are puzzling because, from a strength point of view, how can weaker items leapfrog over stronger items after a single presentation? Even if one argues that low-frequency, or A, items are somehow special or more distinctive than high-frequency, or B, items, such an explanation is only describing the results unless distinctiveness can be independently measured and the learning mechanisms can be specified. Also, this explanation does not apply to the spacing effect where there are no separate A and B items. It has been argued that the leapfrog effect is inconsistent with a strength model for the 


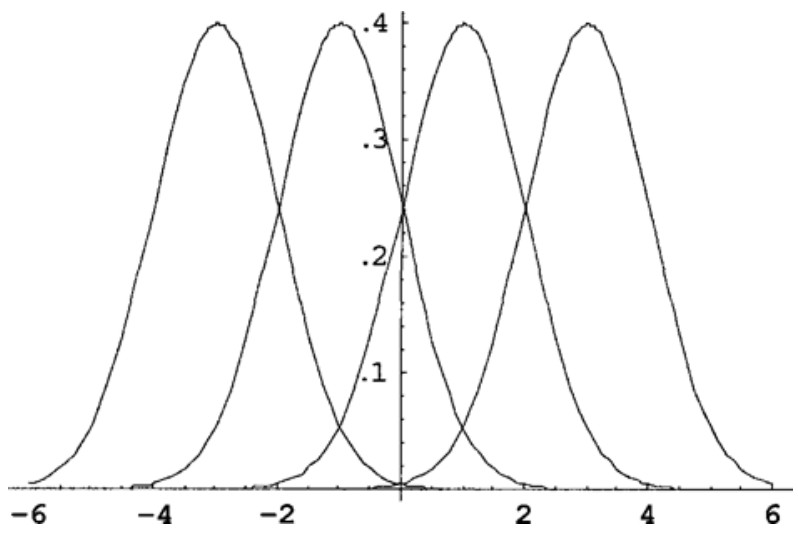

Figure 1. Heuristic distributions for the mirror effect. From left to right, new low-frequency $(\mathrm{AN})$ items, new high-frequency $(\mathrm{BN})$ items, old high-frequency (BO) items, and old low-frequency (AO) items. Redrawn from "The Regularities of Recognition Memory," by M. Glanzer, J. K. Adams, G. J. Iverson, and K. Kim, 1993, Psychological Review, 100, p. 547. Copyright 1993 by the American Psychological Association. Adapted with permission.

mirror effect (Glanzer, Adams, Iverson, \& Kim, 1993), and presumably, the same logic would extend to the spacing effect.

\section{Forced Choice}

There are a variety of formal models (see the Discussion section) that can explain the mirror effect, but in general, they have not been extended to the spacing effect. Also, for the most part, these mirror effect models have dealt only with a yes-no or a confidence judgment procedure and have not been applied to the forced-choice inequalities. ${ }^{1}$ The forced-choice inequalities consist of six pairwise comparisons. The first two comparisons are the null choices; if $\mathrm{N}$ denotes a new item and $\mathrm{O}$ denotes an old item, the first null choice is AN/BN, where the forced choice is between a new A (or low-frequency) item and a new $\mathrm{B}$ (or high-frequency) item. The second null choice is $\mathrm{BO} / \mathrm{AO}$, where the forced-choice is between an old B item and an old A item. ${ }^{2}$

The other four pairwise comparisons can be considered true choices because, in fact, one item is new and the other item is old. The four true choices are $\mathrm{BN} / \mathrm{BO}$, $\mathrm{AN} / \mathrm{BO}, \mathrm{BN} / \mathrm{AO}$, and $\mathrm{AN} / \mathrm{AO}$. In forced-choice tasks, the typical dependent variable is percentage correct. The forced-choice inequalities and the obtained percentage correct from a representative study (Glanzer et al., 1993, Table 7, p. 559) are shown in Table 1. As can be seen by comparing Table 1 with Figure 1, both null choices should be (and are) greater than chance (i.e., .5), and the true choices should be (and are) ordered $2 / 3<1 / 3 \cong 2 / 4<1 / 4$ if the $\mathrm{AN}, \mathrm{BN}, \mathrm{BO}$, and $\mathrm{AO}$ conditions are mapped into the integers $1,2,3$, and $4 .^{3}$

The forced-choice inequalities are much more difficult to model than the simple mirror ordering (AN < $\mathrm{BN}<\mathrm{BO}<\mathrm{AO}$ ). In fact, as I will show shortly, the simple mirror ordering is very easy to model. The main rea- son that the forced-choice inequalities are more difficult is because it is necessary to specify the underlying distributions for $\mathrm{AN}, \mathrm{BN}, \mathrm{BO}$, and $\mathrm{AN}$, and not just the means. Not only must the AN distribution leapfrog over the $\mathrm{BN}$ and the $\mathrm{BO}$ distributions, but also the initial AN and $\mathrm{BN}$ distributions must have the right characteristics to yield the first null choice result (i.e., .60-.65). Of course, one can assume underlying distributions that have this property, but then one has not explained anything; one has just assumed what needs to be explained.

Also, in a sense, one has one degree of freedom, rather than two degrees of freedom. With a yes-no procedure, one can use changes in strength, changes in criterion, or both to fit the data. In forced-choice data, there is no criterion (it is a winner-take-all procedure), so one must rely purely on strength differences to explain the data (Shepard \& Chang, 1963) - and as was noted, not just mean strength, but the full strength distribution, and this is a far more challenging task.

\section{THE EICL MODEL}

The excitation, inhibition, and closed-loop (EICL) model assumes that there are three aspects to strength: an excitatory component, an inhibitory component, and a context component. These are the three components that together determine strength. The excitatory component is viewed as positive strength and will be denoted $x$. The inhibitory component is viewed as negative strength and will be denoted $y$. There is also context strength, denoted by $z$, and the net strength, or $s$, is then the excitatory less the inhibitory plus the context strength, or $s=x-y+z$.

What are these strengths? No one knows, so at this stage of our knowledge they can be thought of as hypothetical constructs. Strength is not unitary; many models, such as SAM (Gillund \& Shiffrin, 1984), MINERVA2 (Hintzman, 1988), TODAM2 (Murdock, 1997), the bind-

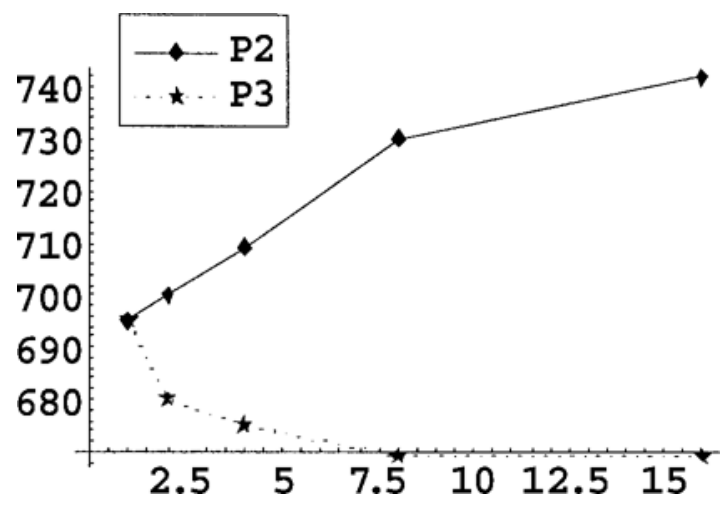

Figure 2. Response times on the second presentation (P2) and the third presentation (P3) as a function of the spacing between the first presentation (P1) and P2. Redrawn from "Recognition Time: Effects of Recency, Frequency, and the Spacing of Repetitions," by D. L. Hintzman, 1969, Journal of Experimental Psychology, 79, p. 194. Copyright 1969 by the American Psychological Association. Adapted with permission. 
Table 1

The Forced-Choice Inequalities for the Accuracy Condition of Table 7 in Glanzer, Adams, Iverson, and Kim (1993)

\begin{tabular}{cc}
\hline Choices & Percentage Correct \\
\hline Null & $.65, .62$ \\
True & $.77, .81, .83, .88$ \\
\hline
\end{tabular}

Note-The null choices are $\mathrm{AN} / \mathrm{BN}$ and $\mathrm{BO} / \mathrm{AO}$; the true choices are (in order) $\mathrm{BN} / \mathrm{BO}, \mathrm{AN} / \mathrm{BO}, \mathrm{BN} / \mathrm{AO}$, and $\mathrm{AN} / \mathrm{AO}$.

cue-decide, or BCD, model (Dennis \& Humphreys, 2001), and TECO (Sikström, 1996, 2000), assume there are two components: item strength and context strength. Here, I am extending the list to three, where the third is inhibitory strength. In various guises, inhibition has also been used in learning and memory models; some of these models will be noted in the Discussion section.

The EICL model also uses a closed-loop learning algorithm (Adams, 1967, pp. 291-293). This is variously called the Rescorla-Wagner, Widrow-Hoff, or delta rule, and the basic idea is simple. On each trial, the learning increment is proportional to the amount yet to be learned, so the closer a strength value is to the asymptote, the smaller the increment. This principle applies to all three components $(x, y$, and $z$ ) separately. There are separate learning-rate constants for each component, and their values vary with the component (excitatory, inhibitory, and context). There are also separate forgetting parameters for each component, so the current strength is the prior strength decremented by a function of the retention interval. Again, the values of these parameters vary with the component. The closed-loop algorithm is an adaptive algorithm, and it avoids the noise saturation problem of open-loop models (Grossberg, 1978). It also can explain some puzzling recognition memory data on the effects of stimulus variation on recognition memory (McDowd $\&$ Murdock, 1986) 4 and was used in TODAM to explain some basic effects in paired-associate learning and retention (Lewandowsky \& Murdock, 1989).

If $x_{j}$ is the excitatory component for the $j$ th presentation of an item and $y_{j}$ the inhibitory component for the $j$ th presentation of an item, $\alpha$ and $\beta$ are the excitatory and inhibitory forgetting parameters, and $a$ and $b$ are the excitatory and inhibitory learning rate constants, the update equations for the excitatory and inhibitory components are

$$
x_{j}=\alpha^{\operatorname{lag}} x_{j-1}+a\left(1-\alpha^{\operatorname{lag}} x_{j-1}\right)
$$

and

$$
y_{j}=\beta^{\text {lag }} y_{j-1}+b\left(1-\beta^{\text {lag }} y_{j-1}\right),
$$

where lag is the number of presentations intervening between presentation $j-1$ and presentation $j$. The first term ( $\alpha^{\operatorname{lag}} x_{j-1}$ or $\left.\beta^{\operatorname{lag}} y_{j-1}\right)$ is the decrement (forgetting), and the second term $\left[a\left(1-\alpha^{\text {lag }} x_{j-1}\right)\right.$ or $\left.b\left(1-\beta^{\operatorname{lag}} y_{j-1}\right)\right]$ is the increment (learning). ${ }^{5}$

All these equations say is that every time other items are presented, the excitatory and inhibitory components of the target item are decremented (by $\alpha$ and $\beta$, respectively), but when the target item itself is presented, its excitatory and inhibitory components are incremented by an amount proportional to the distance from their ceiling, and the proportionality constants are $a$ and $b$, respectively. At the same time, the excitatory and inhibitory components of all the other items are decremented. (The target item is decremented too, but it alone is incremented.) The exponent for $\alpha$ and $\beta$ is lag because the greater the number of intervening items (lag), the more times this decrementing will have occurred.

With this particular version of a closed-loop algorithm, both excitatory and inhibitory components have two factors, a decrement and an increment. The decrement is forgetting from the prior presentation, which depends both on lag and on the values of $\alpha$ (for excitatory) and $\beta$ (for inhibitory). The increment is proportional to the complement of the current strength value, and the proportionality, or learning rate constant, is $a$ (for excitatory) and $b$ (for inhibitory).

\section{Mirror Ordering}

Here is a simple numerical example to illustrate how easy it is to generate the mirror ordering of Figure 1 with the right parameter values. Assume that there are no context effects and $\alpha$ and $\beta$ are so close to 1.0 that their effects can be disregarded. Then all we need are the learning rate constants ( $a$ for excitatory, $b$ for inhibitory) and the starting values for the low-frequency (A) and highfrequency (B) excitatory and inhibitory values (i.e., the values of $x_{j-1}$ and $\left.y_{j-1}\right)$. These are shown in Table 2, along with the predicted result (the mirror ordering). The predicted results are simply $s_{j}$ for the low- and highfrequency words where, in both cases, $s_{j}=x_{j}-y_{j}$ and both $x_{j}$ and $y_{j}$ are obtained from the update equations.

Why do these equations generate a mirror ordering? There is a ceiling effect for the high-frequency items, so the possible increment is smaller for them than for the low-frequency items. Also, the learning rate constant for the inhibitory component $(b)$ is smaller than the learning rate constant for the excitatory component $(a)$, so the ceiling effect has a greater impact on the excitatory com-

Table 2

Hypothetical Learning Rate Constants ( $a$ and $b$ ), Starting Strength Values for Low-Frequency (LF) and High-Frequency (HF) Excitatory and Inhibitory Strengths, and the Mirror Ordering

Learning Rate Constants

$$
a=.2 \quad b=.01
$$

Starting Values

\begin{tabular}{llr} 
& LF & HF \\
$x$ & .2 & .8 \\
$y$ & .05 & .6 \\
\multicolumn{3}{c}{ Mirror Ordering }
\end{tabular}

$.20 \quad .236$


ponent than on the inhibitory component. The net result is a clear mirror ordering where AN jumps over $\mathrm{BN}$ and BO to go from the weakest to the strongest. Note how much larger the increment is for the low-frequency items $(.301-.15=.151)$ than for the high-frequency items $(.236-.20=.036)$.

There are two problems with this example. The first problem is that the starting values were assumed and there are many starting values that will not give the right result (i.e., a mirror ordering). It is necessary to show how the starting values were obtained, ideally from a lexicon (or semantic memory) that the subject brought to the experiment. This lexicon should be learned using the same learning rules and the same parameter values as those used in the study phase (i.e., to update $\mathrm{s}_{j-1}$ to $s_{j}$ ). I will do that here.

The other problem is the forced-choice inequalities. Although it is very easy to produce an example that will generate a mirror ordering, it is much more difficult to get the right forced-choice inequalities, because as was noted, these depend on the distributions of the strength values, not just on the means.

\section{SIMULATION}

\section{Toy Lexicon}

The first step in EICL is to generate a toy lexicon in which the lexical items vary in frequency. As will be discussed below, differences in frequency determine lag, and such a lexicon provides the starting strength values for the model. The toy lexicon is the theoretical counterpart to the empirical approach of prefamiliarizing subjects with a set of items prior to the start of the recognition test (Maddox \& Estes, 1997). I call it a toy lexicon because, obviously, it is a very poor imitation of the actual lexicon that real subjects bring to the experimental session, although one such lexicon has been developed (Kwantes \& Mewhort, 1999). The toy lexicon consists of 1,000 types, which, by Zipf's law (Zipf, 1945), leads to about 7,070 tokens. (Each word is a type, and each presentation of a word is a token.) ${ }^{6}$

Zipf's law states that the frequency of presentation of each word is inversely proportional to its rank, so here the frequencies of the four most common words are (the integer values of ) $1,000 / 1$ or $1,000,1,000 / 2$ or 500 , $1,000 / 3$ or 333 , and $1,000 / 4$ or 250 . This pattern continues for all 1,000 types, and it results (by summation) in a total of 7,069 tokens. The lag is simply the number of tokens divided by the frequency. For the most common item, the lag would be 7,069/1,000 or 7 items intervening between presentations, for the next most common item 7,069/500 or 14 items, 7,069/333 or 21 for the next, and so on.

However, there is a problem when one uses the Zipf rule (or any other differential frequency rule) for frequency of presentation. The problem is the AN/BN null choice. If rank determines frequency and frequency de- termines strength and we are comparing low-frequency (A) items with high-frequency (B) items, there will not be enough overlap of $\mathrm{AN}$ and $\mathrm{BN}$. To fit the data, we need enough overlap to produce a forced-choice probability correct of about .60-.65. How can this happen?

We can solve this problem (and also make the model more realistic) if we add parameter variability. Both number of presentations and lags must be random variables whose means are determined by the rank frequency, but there is some variability. To illustrate, item rank 3 will have a mean number of presentations of 333 and a mean lag of 7,069/333 $=21.23$ or 21 items. Item rank 4 will have a mean number of presentations of 250 and a mean lag of $7,069 / 250=28.28$ or 28 items. Then, 21 is the mean lag for the rank 3 item, and 28 is the mean lag for the rank 4 item, but the actual lag in forming the lexicon was a random sample from a normal distribution with means of 23 and 28 , respectively. ${ }^{7}$

Since the number of presentations and the lags are random samples from truncated normal distributions, it is necessary to specify the standard deviations of these normal distributions. In each case, I used the coefficient of variation $V$ to determine the standard deviation. (If $\mu$ is the mean, then $V=S D / \mu$, so $S D=V \mu$.) For the betweenlag variation, if the rank 3 item happened to be presented 21 times in forming the lexicon, then this too was a random variable with $S D=V \mu$, where $\mu=21$. Since simulations of random permutations of relatively small numbers of items whose frequency was determined by Zipf's law showed that, in general, the $S D$ was about equal to the mean, I set $V$ to 1.0 for both the between- and the within-lag variabilities.

I assumed a list length of 41 items (i.e., $41 \mathrm{~A}$ items and $41 \mathrm{~B}$ items), and the ranks were 130-170 for the highfrequency, or B, items and 830-870 for the low-frequency, or A, items. It is not necessary to form the entire lexicon; all that is necessary is to determine the strength of $41 \mathrm{~A}$ items and $41 \mathrm{~B}$ items with the appropriate frequencies and lags, using the update equations given above. One needs the forgetting parameters $\alpha$ and $\beta$ and the learning rate parameters $a$ and $b$ for the excitation and the inhibition, and these too (i.e., $\alpha, \beta, a$, and $b$ ) were random variables. The lexicon simulation consisted of forming two four-way type (new or old) $\times$ rank frequency (low [A] or high [B] $) \times$ subjects $(500) \times$ items $(41)$ arrays - one for excitation $(x)$ and the other for inhibition $(y)$. Context will come in later.

In the simulation, since the values of $\alpha, \beta, a$, and $b$ were random variables, the actual values used in the simulations varied from subject to subject, as well as according to type (new or old) and rank (low or high). However, they were fixed for all items learned by that subject in that condition. The mean forgetting parameter values were $\alpha=.99995$ and $\beta=.99999$, both with $V=$ .00005 . The mean learning rate values were $a=.08$ and $b=.008$, both with $V=.10$. It may come as a surprise to see five-place accuracy in the parameter values, but 
with the long lags that are encountered in learning a lexicon, we are in quite a different part of the parameter space than in episodic memory tasks.

As will be shown, these parameter values gave reasonable results for the AN/BN forced-choice inequality. Given the enormity of the parameter space, I attempted only a rather cursory investigation of parameter sensitivity (Li, Lewandowsky, \& DeBrunner, 1996), and the results will be given later (Table 7).

Study phase. The first problem, then, was to find the right parameter values to generate a lexicon with the right value of AN/BN (new low-frequency to new highfrequency). Having achieved this, the other main problem was to generate a leapfrog effect for the distributions that would give the remaining forced-choice inequalities. The general solution was to add a third component, context, to the net strength. Thus, as was mentioned above, for net strength, $s_{j}=x_{j}-y_{j}+z_{j}$, where $z_{j}$ is the current context strength.

The complete set of update equations for $x$ (excitatory), $y$ (inhibitory), and $z$ (context) is shown in Table 3 . The update equation for context is different from those for excitation and inhibition, in that it depends not only on the prior context strength $\left(z_{j-1}\right)$, but also on the prior excitatory strength $\left(x_{j-1}\right)$. The fact that it is different should not be surprising, because if it were identical, it would be redundant, and one could model it with a weighting parameter on $x$ (or $x-y$ ). The way it is different is what is crucial. The first and third equations are coupled because $z_{j}$ depends both on $z_{j-1}$ and $x_{j-1}$. With this coupling, it is possible to produce the leapfrog effect, whereas it cannot be done with just a simple closed-loop algorithm.

The forgetting parameter for context is $\gamma$ (gamma, not to be confused with $y$ ), and the context learning rate constant is $c$. All the parameter values are shown in Table 4. The forgetting parameter for context $(\gamma)$ is lower, so the forgetting is faster than the forgetting rate for excitation $(x)$ and inhibition $(y)$. Also, the learning rate constant $(c)$ is larger than the learning rate constants for excitation and inhibition. This is because context is assumed to be more labile than excitation and inhibition. ${ }^{8}$

In the simulation, after the lexicon was formed, the excitatory and inhibitory components of the new items ( $\mathrm{AN}$ and $\mathrm{BN}$ ) were decremented by their forgetting parameters at a random lag appropriate for their frequency.

Table 3

The EICL Update Equations for the Mirror Effect

\begin{tabular}{|c|c|c|c|}
\hline \multirow[b]{4}{*}{ Strengths } & \multirow{2}{*}{\multicolumn{2}{|c|}{$\begin{array}{l}x_{j}=\alpha^{\mathrm{lag}} x_{j-1}+a\left(1-\alpha^{\mathrm{lag}} x_{j-1}\right) \\
y_{j}=\beta^{\mathrm{lag}} y_{j-1}+b\left(1-\beta^{\mathrm{lag}} y_{j-1}\right) \\
z_{j}=\left(1-\gamma^{\mathrm{lag}}\right) z_{j-1}+c\left(1-\alpha^{\mathrm{lag}} x_{j-1}\right)\end{array}$}} & \multirow[b]{3}{*}{ Context } \\
\hline & & & \\
\hline & Excitatory & Inhibitory & \\
\hline & $x$ & $y$ & $z$ \\
\hline Forgetting & $\alpha$ & $\beta$ & $\gamma$ \\
\hline Learning & $a$ & $b$ & $c$ \\
\hline
\end{tabular}

Table 4

Parameter Values for the Update Equations of Table 3

\begin{tabular}{lll}
\hline \multicolumn{1}{c}{ Parameter } & \multicolumn{1}{c}{ Mean } & \multicolumn{1}{c}{$V$} \\
\hline No. presentations/item & No. tokens/rank & 1.33 \\
Lag & Rank & 1.0 \\
$\alpha$ & .99995 & .00005 \\
$\beta$ & .99999 & .00005 \\
$\gamma$ & .97 & .1 \\
$a$ & .08 & .1 \\
$b$ & .008 & .1 \\
$c$ & .4 & .1 \\
\hline
\end{tabular}

Note $-\alpha, \beta$, and $\gamma$ are the forgetting parameters, and $a, b$, and $c$ are the learning-rate parameters for excitation, inhibition, and context, respectively. $V$ is the coeff icient of variation $(V=S D /$ mean $)$.

This corresponded to whatever forgetting occurred between their last preexperimental presentation and the study phase of the experiment. To simulate the study phase, the excitatory and inhibitory components of the old items were updated according to the equations given above (Table 3 ). It was assumed that there was no overlap between the preexperimental and the experimental contexts, so $z_{j-1}$ was assumed to be zero. Thus, here the context strength $\left(z_{j}\right)$ resulting from the study phase was dependent only on the value of $x_{j-1}$, the strength of the excitatory component at the time of test.

Study-test interference effects were not included in the simulation. The justification was that, with the values of the excitatory and the inhibitory forgetting parameters so close to 1.0, they would have little or no effect on the simulation. However, the situation will change when we come to the spacing effect. Further discussion of this point will be deferred until then.

Test phase. After the study phase, all six forced-choice probabilities were computed on a subject-by-subjectbasis and then averaged. The usual forced-choice algorithm was used, with one slight modification. It seems to be generally assumed that forced choice is scored as correct no matter how small the difference between the stronger and the weaker items. In other words, a step function is used to map the old-new difference into probability correct. This seemed unrealistic, so I used an ogival mapping function. That is, if the strength difference was exactly zero, the probability correct for that item for that subject was assumed to be .5 , and the probability increased with the difference. The mapping function was a cumulative normal distribution with an $S D$ of $.10 .^{9}$

\section{Results}

For the results, the four distributions are shown in Figure 3. For each distribution, the data were pooled over the 500 subjects and 41 high-frequency and low-frequency items and then were tallied in 20 bins from 0 to 1.0 in steps of .05. The four distributions are negatively skewed and become more leptokurtic as they increase in strength. They are somewhat different from the normal or the binomial distributions assumed in some models of the mirror effect.

The ordering of these distributions shows a mirror effect, but what produced it? The difference in word frequency determined lag, and the lag (by the update equa- 


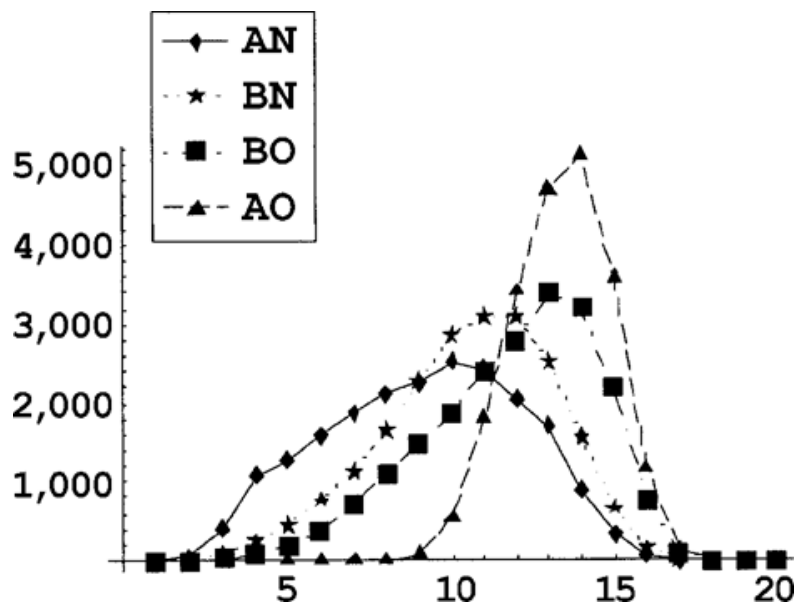

Figure 3. Frequency distributions from an EICL simulation of the mirror effect showing the $\mathrm{AN}, \mathrm{BN}, \mathrm{BO}$, and $\mathrm{AO}$ strength distributions pooled over subjects and items from a simulation with 500 subjects and a list length of 41 for both the $A$ and the $B$ items. The parameter values used are shown in Table 4. The horizontal axis is strength $(x-y+z)$ in 20 steps from .025 to .975 , and the vertical axis is frequency.

tions) determined strength. Thus, no parameters differed for low and high frequencies, and the underlying distributions were derived, not assumed.

The means and $S D$ s of each distribution are shown in Table 5. The means show a perfect mirror order (including a sizable leapfrog effect), so the means substantiate our claim about the mirror ordering. The $S D$ s are about the same, except that the $S D$ for AO is about half the $S D$ for AN. We know from studies of receiver-operating characteristics (ROC) curves in recognition memory that the old-item variance as inferred from ROC slopes is generally larger than the new-item variance (Glanzer, Kim, Hilford, \& Adams, 1999; Ratcliff, Sheu, \& Gronlund, 1992), and this effect was not obtained here. This problem will be considered at the start of the Discussion section.

The forced-choice inequalities are also shown in Table 5; these are means over subjects. The null choices are in the low .60s, and the true choices are ordered exactly as they should be. Thus, EICL can generate the right results for the mirror effect. With one exception (the $\mathrm{AN} / \mathrm{AO}$ variances), the results seem reasonably close to the actual data; the mirror ordering was obtained, and the forced-choice inequalities showed the right pattern.

To reduce the number of free parameters slightly and check on the reliability of the results, the simulations were rerun with $\alpha=\beta$ and 500, 1,000, and 2,000 replications (subjects). The mirror ordering and the forcedchoice inequalities for this six-parameter model are shown in Table 6, and they clearly replicate the results in Table 5. Also, reliability is not a problem, since the results for $N=500,1,000$, and 2,000 are essentially indistinguishable.

Why does EICL predict the forced-choice inequalities? Unlike for the mirror ordering, there is no simple answer.
For the forced-choice inequalities, you seem to need all three components of the model: excitation and inhibition, the closed-loop algorithm, and parameter variability (random variables). As far as I can tell, no two alone will suffice. Without parameter variability, the AN/BN comparison would be too high, even with excitation/inhibition and the closed-loop algorithm. Then (as with the mirror ordering), you need excitation, inhibition, and the closedloop algorithm to get the leapfrog effect. And of course, even then you have to have the right parameter values and the right set of difference equations. So the forcedchoice equalities depend not only on parameter variability, excitation/inhibition, and the closed-loop algorithm, but also on the right parameter values and the difference equations.

To see what effect lag variability had on the AN/BN forced-choice comparison, I ran a small grid search where both $V_{\text {rep }}$ (the coefficient of variation $V$ for repetition) and $V_{\text {lag }}$ (the coefficient of variation $V$ for the lag between items) were $0.1,0.4,0.7,1.0$, and $1.3($ crossed $5 \times 5$ ) with 100 subjects (replications) each. The results are shown in Table 7, where the mean percentage correct is shown as a joint function of $V_{\text {rep }}$ (rows) and $V_{\text {lag }}$ (columns). As is obvious by inspection, repetition variability had a large effect, but variability in item lags had essentially no effect.

\section{SPACING EFFECT}

The basic spacing effect refers to the fact that "a piece of information studied on four occasions widely spaced apart in time will be remembered better than a similar fact studied on four occasions closer to each other. This is most commonly known as the spacing effect" (Greene, 1992, p. 145). However, the situation is more complicated than this, since it depends on the intertrial interval (between, say, the first two presentations), the retention interval (following the second presentation), and the type of task (item recognition, associative recognition or recall, or free recall; Haberlandt, 1999). There is the further complication that immediate repetition often has no benefit, repetition at short intervals is actually better at short retention intervals, but spaced repetition is better at long retention intervals.

The immediate-repetition effect could be an attention effect and will not be considered here. The focus is on item recognition, so the paired-associates and free-recall

Table 5

Means and $S D$ s of the Four Distributions From the EICL Simulation Pooled Over Subjects and Items and the Resulting Mean Forced-Choice Inequalities (Means Over Subjects)

\begin{tabular}{lllll} 
& \multicolumn{4}{c}{ Distributions } \\
\cline { 2 - 5 } Mean & AN & BN & BO & AO \\
\cline { 2 - 5 }$S D$ & .43 & .50 & .57 & .64 \\
& .148 & .127 & .127 & .073 \\
& \multicolumn{5}{c}{ Inequalities } \\
\hline
\end{tabular}

\begin{tabular}{llllll}
\hline $\mathrm{AN} / \mathrm{BN}$ & $\mathrm{BO} / \mathrm{AO}$ & $\mathrm{BN} / \mathrm{BO}$ & $\mathrm{AN} / \mathrm{BO}$ & $\mathrm{BN} / \mathrm{AO}$ & $\mathrm{AN} / \mathrm{AN}$ \\
\hline
\end{tabular}

$\begin{array}{llllll}.603 & .655 & .610 & .695 & .765 & .832\end{array}$


Table 6

Mirror Ordering and Forced-Choice Inequalities for Three Values of $N$ (Number of Subjects) With the Following Parameter Values: $\alpha=.99997, \gamma=.97, a=.08, c=.4$, $V_{\alpha}=.00005$, and $V_{\gamma}=.1$. Then $\beta=\alpha, b=a / 10, V_{\beta}=V_{\alpha}$, and $V_{a}=V_{b}=V_{c}=V$.

\begin{tabular}{rllll}
\hline \multicolumn{5}{c}{ Mirror Order } \\
\hline$N$ & AN & BN & BO & AO \\
\hline 500 & .441 & .514 & .578 & .647 \\
1,000 & .453 & .521 & .583 & .654 \\
2,000 & .447 & .519 & .582 & .650
\end{tabular}

Forced-Choice Inequalities

\begin{tabular}{cccccc}
\hline AN/BN & BO/AO & BN/BO & AN/BO & BN/AO & AN/AN \\
\hline .638 & .646 & .638 & .743 & .791 & .871 \\
.628 & .647 & .633 & .731 & .787 & .864 \\
.637 & .643 & .635 & .740 & .785 & .867 \\
\hline
\end{tabular}

results will not be considered either. Then, for two presentations, the spacing effect can be seen as a bivariate function of the P1-P2 (interpresentation) interval and the P2-test (retention) interval. EICL can, in principle, model the full bivariate function, but we will illustrate only with selected $\mathrm{P} 1-\mathrm{P} 2$ intervals. To the extent that the model can adequately represent these functions, we can say that it captures the effects of spacing on the rate of forgetting.

\section{Simulation}

The spacing effect was simulated in the same general way as the mirror effect, but there were a few differences. There were no separate A and B items; the rank frequencies of the items ran from 230 to 270 . It seemed reasonable to assume that studies of the spacing effect (of which there are many) typically use common words, but not necessarily high-frequency words. There were no new items; following the lexicon learning, all items were presented twice. The interval (number of presentations) between the first and the second presentations is called lag 1, and the interval between the second presentation and the test is called lag2.

In other respects (including update equations, parameter values, and use of random variables), the simulation was identical to that used for the mirror effect. The simulations were based on 500 subjects and 41 items. The update equations for $x_{j}, y_{j}$, and $z_{j}$ are shown in Table 8 , and they are a function of lag2. The values of the excitatory, inhibitory, and context strength after the time of test are denoted as $x_{j+1}, y_{j+1}$, and $z_{j+1}$, and the equations in Table 3 take $x_{j-1}, y_{j-1}$, and $z_{j-1}$ to $x_{j}, y_{j}$, and $z_{j}$.

For latency, we computed the appropriate strength values for each subject and each item and then assumed that latency is inversely proportional to strength. The mean latencies (i.e., reciprocal strength values) for P2 and P3 for lags 10, 20, 30, 40, and 50 are shown in Figure 4. These results should be compared with those in Figure 2. Obviously, EICL generates the right pattern of results, both for P2 (forgetting as a function of the P1-P2 lag) and for P3 (learning as a function of the P1-P2 lag).
For simplicity of exposition, I have described this simulation as if it resulted when exactly the same parameter values that had been used for the mirror effect were used in the expressions for the spacing effect. This is essentially correct, but there was some very slight jiggling of parameters back and forth to see whether one could improve the spacing results without damaging the mirror results. ${ }^{10}$ Also, several other update equations for context that could generate a mirror effect were rejected when it was found that they generated a negative spacing effect (poorer retention as spacing increased). ${ }^{11}$

\section{DISCUSSION}

Although EICL can generate most of the right patterns for the mirror effect and the spacing effect, it is off in a few details. The old-item variance is less than the new-item variance for the low-frequency (A) item, and whereas the true choices show the mirror ordering, the $\mathrm{BN} / \mathrm{BO}$ comparison is lower than it should be.

The $S D$ for AO for the mirror effect is about half that for $\mathrm{AN}$ and this is probably wrong. ${ }^{12}$ Why is it off? The most likely possibility is that there is no study-test interference. In terms of TODAM2, this means that there is no chance for context from other list items to affect the comparison of the probe and the memory vectors. Given context drift, this is sure to increase the old-item variance (for both A and B items); after all, this is the whole point of global-matching models. Consequently, it is not possible that EICL, as it stands, can capture the effect of interitem context similarity. Including study-test interference in EICL might solve this problem. The same comments apply to the $\mathrm{BN} / \mathrm{BO}$ comparison.

The results for the spacing effect are only a very rough fit to any particular set of data. However, they show the right pattern, and this in itself was not easy to achieve. Moreover, these plots result from a simulation using the same update equations (Table 3 ) and parameter values (Table 4) as the simulations for the mirror effect. There were no ad hoc parameters that varied with lag. Thus, as with the mirror effect, the model, not the parameters, was doing the work.

\section{Other Mirror Results}

In addition to the mirror ordering and the forced-choice inequalities, there are other mirror effects that have been reported. One of them is concentering. Concentering refers to the fact that the forced-choice distributions are less widely separated for a delayed test than for an im-

Table 7

Mean AN/BN Percentage Correct Forced Choice as a Joint Function of $V_{\text {rep }}$ (Rows) and $V_{\text {lag }}$ (Columns)

\begin{tabular}{lllll}
.958 & .958 & .955 & .951 & .945 \\
.876 & .871 & .869 & .866 & .863 \\
.732 & .735 & .729 & .725 & .715 \\
.608 & .602 & .614 & .608 & .603 \\
.505 & .511 & .512 & .504 & .494 \\
\hline
\end{tabular}


Table 8

Update Equations for the Spacing Effect

$$
\begin{aligned}
& x_{j+1}=\alpha^{\operatorname{lag} 2} x_{j} \\
& y_{j+1}=\beta^{\operatorname{lag} 2} y_{j} \\
& z_{j+1}=\gamma^{\operatorname{lag} 2} z_{j}
\end{aligned}
$$

Note $-x_{j+1}, y_{j+1}$, and $z_{j+1}$ are the excitatory, inhibitory, and context strength at the time of test, and lag2 is the retention interval (number of intervening items) between the second presentation and the test.

mediate test but the mirror ordering is preserved. This could come about if, as is shown in Figure 1, the four distributions were centered on the midpoint between $\mathrm{BN}$ and BO but, with delay, they all moved closer to this midpoint.

To see if EICL could capture this effect, an additional step was added to the simulation. The excitation, inhibition, and context values for the A and B items that would have been used for an immediate test were decremented by the mean values of the forgetting parameters before the forced-choice inequalities were computed. A delay of 20 intervening items was assumed. A delay of 20 was assumed because, with that value, the context would have dropped to about half of what its value would have been on an immediate test.

The resulting forced-choice inequalities were .603 and .523 for the null choices and $.559, .654, .582$, and .679 for the true choices, and these values may be compared with the forced-choice results from the immediate test that are shown in Table 5. The right pattern of results was found, even though it was not a particularly good fit. One aspect of the concentering results that is of particular significance (Glanzer, Adams, \& Iverson, 1991) is the fact that the AN/BN inequality decreases from an immediate to a delayed test. As these authors noted, according to a strength theory, there is no reason for this to occur, and in this simulation, it was so small it would probably be undetectable in real data.

However, this small effect may be related to the fact that, in the simulations, I assumed there was no context similarity between preexperimental and experimental contexts, and this may be wrong. I could have assumed the opposite, but that would have made the simulation even more complex. That is, if one assumed that there was some context similarity between the preexperimental and the experimental situations, one would also have to allow the possibility that study items occurred during the delay. Suffice it to say that this possibility would have necessitated a completely different approach in the simulation and, with a reasonably sized lexicon, would have been too time consuming to be feasible.

Another interesting result is that multiple presentations of the high-frequency (B) items in the study phase can affect the mirror ordering because, then, $\mathrm{BO}$ may be greater than AO (Sikström, 2001; Stretch \& Wixted, 1998). I ran a simpler version in which the update equations were run on the mean values but there was no variability. The results (AN, BN, BO, and $\mathrm{AO}$ ) were $.104, .317, .612$, and
.519 for the control (1P) condition and .104, .317, .675, and .519 for the $5 \mathrm{P}$ condition. With this simpler version, we did not get the ordinary mirror effect for the $1 \mathrm{P}$ control condition $(\mathrm{BO}>\mathrm{AO})$ but the $\mathrm{BO}-\mathrm{AO}$ difference increased, as it did in Stretch and Wixted's result.

Why did we not get the mirror order in the control condition? There are at least three (not necessarily mutually exclusive) reasons: (1) There was no variability, (2) there was no study/test input/output interference, and (3) the raw lexicon frequencies were very small (2 for the A items, 7 for the B items). The low lexicon frequencies require explanation.

The low lexicon frequencies are a consequence of the Zipf rank-frequency distribution. With 1,000 types, the raw frequency of item rank 1 is 1,000 ; that for rank 2 is 500 ; for rank 3 , it is 333 ; and so on, so that by the time you are down to rank 150 (the rank of the B items) the frequency is, as was noted, only 7 (rounding up) and for rank 850 (the rank of the $\mathrm{A}$ items) is only 2 (rounding up). The AN/BN difference was very large, so the leapfrog effect does not reverse things. But the increase for the $\mathrm{B}$ items in the $5 \mathrm{P}$ condition is larger in the experimental (5P) condition, and this is the main point of Stretch and Wixted's (1998) result. And as before, the same parameter values were used in both the lexicon learning and the experimental sessions.

It should be kept in mind that the goal of EICL is to generate data that matches the patterns of the mirror and the spacing effects. The goal is not to fit the data. The parameter space is enormous, and the chance of finding exactly the right parameter values to fit the data, even if such values do exist, is very small. This is the advantage of analytic models, such as attention-likelihood theory and TODAM2. However, there are important models (e.g., SAM and MINERVA) that are basically simulation models that, for the most part, rely on pattern matches.

To give some indication of what learning in EICL is like, I ran the simpler version (i.e., no variability) for the $\mathrm{B}$ items for five trials and recorded the $x, y$, and $z$ values

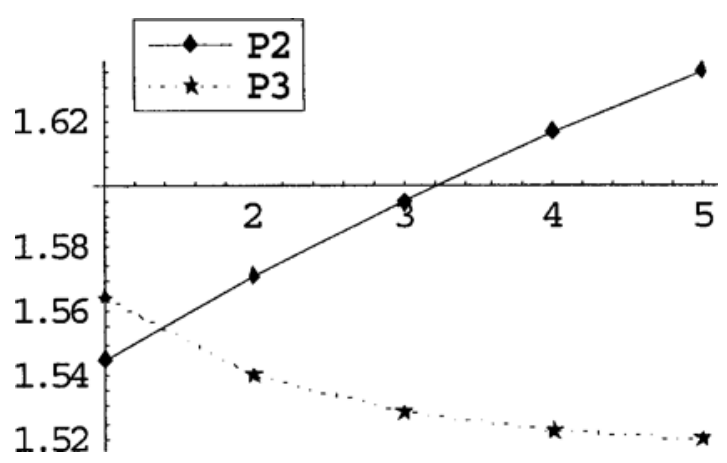

Figure 4. Results from an EICL simulation of the spacing effect showing latencies (i.e., reciprocal strengths) on the second presentation $(\mathrm{P} 2)$ and the third presentation $(\mathrm{P} 3)$ as a function of the lag in units of 10 between the first presentation (P1) and the second presentation (P2). That is, " 2 " on the horizontal axis of the graph is lag 20, " 3 " is lag 30 , and so on. 
Table 9

Trial-by-Trial Excitation $(x)$, Inhibition $(y)$, Context $(z)$, and Net $(s)$ Strengths for Five Presentations (P) of the High-Frequency $B$ Items

\begin{tabular}{ccccc}
\hline $\mathrm{P}$ & $x$ & $y$ & $z$ & $s$ \\
\hline 1 & .419734 & .060119 & .252289 & .612 \\
2 & .466155 & .067638 & .232106 & .631 \\
3 & .508863 & .075096 & .213538 & .647 \\
4 & .548154 & .082496 & .196455 & .662 \\
5 & .584302 & .089836 & .180738 & .675 \\
\hline
\end{tabular}

Note-The starting values came from the lexicon, and the parameters were as shown in Table 4, except that there was no variability.

separately. These are shown in Table 9, along with the strengths $(s=x-y+z)$. As can be seen, both excitatory $(x)$ and inhibitory $(y)$ strengths increased, but context $(z)$ actually decreased. However, the net strength $(s)$ does increase, and this is why EICL can predict that you do not get a mirror effect with repetition of the high-frequency B items (Stretch \& Wixted, 1998).

\section{Inhibition}

Probably the most novel (or contentious)innovation of EICL is the assumption of inhibition. However, this idea is not new. In an early model of paired-associate learning (Thurstone, 1930), it was assumed that practice served two functions: strengthening of the correct response (excitation) and weakening of the incorrect responses (inhibition). It was possible to derive various predictions about pairedassociate learning (in particular, the length-difficulty relation) by solving the pair of differential equations that were postulated. ${ }^{13}$ Of course, this is not quite the same as EICL, because inhibition operated on competitors, whereas here, excitation and inhibition are assumed to be two components of the same memorial representation.

Perhaps a closer parallel is the approach/avoidance paradigm of animal behavior (Townsend \& Busemeyer, 1978), which followed an analysis of discrimination learning based on an excitatory tendency of the positive stimulus and an inhibitory tendency of the negative stimulus (Spence, 1937). For an approach/avoidance conflict, the same goal (e.g., a food box) can have both attractive properties (leading to approach) and aversive properties (leading to avoidance). The avoidance gradient has a steeper slope, but the curves cross. If the animal is too close it will back up, but if it is too far away it will approach, and the gradients are such that the animal is predicted to vacillate around some point of stable equilibrium. Approach and avoidance are terms that describe the behavior of an animal, but these notions could clearly be rephrased in terms of excitatory and inhibitory tendencies, and here the excitatory and inhibitory tendencies are operating on the same underlying processnamely, the tendency to approach the goal box.

We have probably all had comparable experiences, both with spatial and temporal dimensions. Watch tourists at the top of the Grand Canyon: They edge closer to get a better view, but they back up when they get too close. We are asked to give a talk or serve on a committee some time in the future; it seems like a good idea (or at least we are willing) at the time, but as the deadline approaches we sometimes wish we had not said "yes." Not only are "excitation" and "inhibition" acting on the same behavior, but in both cases the inhibitory gradient starts lower (so we approach or say "yes"), but with distance or time, the inhibition increases more rapidly than the excitation, so we back off or try to find a good excuse. The same is true in EICL, but the functions are not linear.

Opponent process models, both in vision (Hurvich \& Jameson, 1974) and in motivation (Solomon, 1980), are obvious parallels. Neuroscience is full of so many instances of inhibitory processes that they are accepted as a fact of life. There seem to be excitatory (glutamate) and inhibitory (GABA) synapses in the brain (Kolb \& Whishaw, 1996, p. 84). Also, there is a vast literature dealing with GABAergic inhibitory mechanisms in the hippocampal pyramidal cells. But what about memory?

Habituation reflects a very simple kind of memory, and inhibition plays an essential role in some habituation models (Groves \& Thompson, 1970; Staddon, 1993; Staddon \& Higa, 1996). In the Staddon model, the (excitatory) strength of the current stimulus is attenuated by the (inhibitory) memory for recent experiences with the stimulus. With multiple time scales, it is possible to account for much rate-sensitive data in the nematode. ${ }^{14}$ The model can also be applied to the partial-reinforcement effect and the successive negative contrast effect in animal learning. These principles have also been implemented in a connectionist model with applications to free recall (Sikström, 2003).

Many models of memory (e.g., Bower, 1971; Estes, 1972; Lewandowsky, 1999) have used inhibition one way or another. At the cognitive level, the concept of suppression is widely used in connected discourse (Radvansky, 1999), and surely, at a general level, suppression and inhibition are indistinguishable. Three processes (short-term, long-term, and very long term expectations) are needed to account for the dynamics of operant conditioning (Dragoi \& Staddon, 1999), and mutually inhibitory connections are also an important part of the model (Dragoi \& Staddon, 1999, Figure 1, p. 22).

Strong support for the notion of inhibition can be found in a recent review article reporting several decades of work on memory and learning in simple organisms (Kandel, 2001). Apparently, there are excitatory and inhibitory interneurons in the gill withdrawal reflex of Aplysia (Kandel, 2001, Figure 2C, p. 1032). To quote from a section on inhibitory constraints, "positive regulators are only half the story-there are inhibitory constraints on memory. Long-term synaptic facilitation requires not only activation of memory-enhancing genes, but also inactivation of memory suppressor genes . . . . Thus, during long-term memory storage, a tightly controlled cascade of gene activation is switched on, with memory-suppressor genes providing a threshold or checkpoint for memory storage" (p. 1034). ${ }^{15}$ 
Inhibition is an important component of two recent models for recognition memory, subjective-likelihood (SL) theory (McClelland \& Chappell, 1998) and the retrieving effectively from memory, or REM, model (Shiffrin \& Steyvers, 1997). They will be discussed in the next section. In a more reductionistic vein, another way of modeling inhibition in memory is by a sigmoidal (logistic) activation function with a negative bias (Li, Lindenberger, \& Frensch, 2000): "Small increments in excitatory signals produce greater changes in firing frequency than those produced by the same amount of increment in inhibitory signals" (p. 880). Cognitive effects in paired-associate learning were modeled in "two groups of identical networks that differed only [italics theirs] in their mean $G$ s (one of the parameters of the logistic activation function)." As in EICL, this parameter was treated as a random variable.

As Li et al. (2000) say, reducing mean $G$ reduces the unit's average responsivity and increases the variability in the unit's output activation. They then use this approach to link catecholaminergic modulation with behavioral data. In particular, they model age effects in mean trials to criterion, susceptibility to proactive inhibition, complexity (list length), and intra- and intersubject variability. As in EICL, the nature of the inhibition is not explicitly specified, but its effects are captured in the characteristics of the activation function ( $\mathrm{Li}$ et al., 2000, Figure 2A, p. 881).

In another computational model (Hasselmo \& Wyble, 1997), "in an attempt to link effects at a cellular level to effects at a behavioral level, recognition and free recall were modeled in a computation model of the hippocampus" (p. 2). "It was assumed that each subregion contains interacting populations of excitatory and inhibitory neurons .... This model did not place an artificial constraint on the total output of a neuron .... Total network activity was regulated by feedback from inhibitory interneurons .... A balance of excitatory and inhibitory neurons allowed neurons to enter an attractor state with intermediate levels of activity" (p. 9).

One of the reviewers of the present paper asked "What is being inhibited?' The excitatory component is being inhibited (reduced) by the inhibitory component, and the (algebraic) sum of excitation, inhibition, and context determine the strength of the response. The strength of the response (as in signal detection theory) is fed into a decision process, and for the spacing effect, the old or new response is a function of memory and decision. For forced choice, two such "signals" $(x-y+z)$ are compared on a winner-(generally)-takes-all basis. ${ }^{16}$ The ideas are essentially identical to the approach/avoidance notions, except that the equilibrium concept is not relevant and the mechanisms could be anything from catecholinergic modulation to interneurons to separate excitatory and inhibitory neurons. It seems pretentious even to speculate as to what excitation and inhibition in EICL refer to when detailed reductionist models such as these exist, but there certainly is ample precedent for assum- ing that some sort of inhibitory process contributes to performance in human episodic memory tasks. ${ }^{17}$

\section{Other Models}

Attention-likelihood theory. The first major model of the mirror effect is the attention-likelihood theory, or ALT (Glanzer et al., 1993). ALT is a more elegant and sophisticated model than EICL; it is a formal (analytic) model with many quantitative solutions, it applies to other variables than word frequency, and it can predict the three regularities of recognition memory (the forcedchoice inequalities, the slope regularities, and concentering). However, there are some theoretical issues that raise problems, and experimental studies have raised doubts about the decision rules.

From a theoretical perspective, there are two main problems with ALT, and they have to do with the underlying assumptions. First, it is assumed that low-frequency A items and high-frequency $B$ items come with the same number of marked features; hence, the AN and BN strength distributions are identical. But surely, lowfrequency and high-frequency new items must differ somehow; otherwise, how can one explain differences in such tasks as naming latency and lexical decision? Furthermore, if new A and B items are of equal strength, why should subjects pay more attention to A (low-frequency) items? One could say they are more distinctive, but this is ad hoc.

Second, and perhaps more critical, it is assumed that subjects pay more attention to (mark more features of) low-frequency A items than high-frequency B items. In the model, the attention (or encoding) parameter is greater for the A items than for the B items. So, if it is assumed that new A and B items have the same number of marked features, that subjects attend to (or mark) more features for old A items than for old B items, and that subjects use a log-likelihood decision rule, ALT can show very impressive fits to the data. To me, these assumptions (particularly the first two) mean that the model basically is describing the data, not explaining them. As I have pointed out, in EICL there are no parameters (or processes) that differ for low-frequency and high-frequency items. ${ }^{18}$ I shall return to the point about decision rules shortly. ${ }^{19}$

Subjective-likelihood model. The general ideas behind the SL model (McClelland \& Chappell, 1998) are as follows. Items or events in the real world are represented by binary features. If the feature is present, the representation is 1 ; if absent, it is 0 . The representation of these items have corresponding elements in hypothetical memory vectors that start initially at about .5 , but with experience (repeated presentation), those corresponding to the present features approach a value of 1 , and those corresponding to the absent features approach a value of 0 . This is differentiation, but it could just as well be called excitation and inhibition. At an abstract level, excitation increases or adds, whereas inhibition 
decreases or subtracts. The details of the representation in EICL are somewhat different from the SL model, but conceptually the ideas seem identical.

Item detectors estimate feature probabilities, and these detectors are used to compute SL estimates that gradually differentiate with learning. With differentiation, each detector becomes better at rejecting other items (McClelland \& Chappell, 1998, pp. 748-749). Estimates of the starting (generic) values and likelihoods (products of these probability values) are stochastic. Thus, item detectors learn the best estimates of conditional probabilities of the item features. Bayesian hypothesis testing is used; the subject is assumed to compute a log-odds ratio as the basis for decision. ${ }^{20}$

As in EICL, the learning mechanism is closed loop (approaching 1 or 0 as upper or lower limits), but the learning rate constant $(\epsilon)$ decreases over trials. In particular, $\epsilon$ is inversely proportional to the number of presentations of an item (McClelland \& Chappell, 1998, Equation 8, p. 737). Although this has some desirable statistical properties (p. 737), it would seem to mean that the subject would have to estimate both the current probability values and the number of presentations the item has already had in order to implement the update equation (Equation 7, p. 737). There is no mention of forgetting or context, and the lexicon is assumed to be a tabula rasa.

For the word frequency effect, it is assumed that representations of low-frequency items are less variable than representations of high-frequency items (McClelland \& Chappell, 1998, p. 743). This results from setting a parameter $\left(p_{0}\right.$, the probability of being active for the lowprobability elements of an item) to a higher value for high-frequency items. The justification in the SL model is that there are fewer definitions for low-frequency words than for high-frequency items. There are also different learning-rate constants for high-frequency and low-frequency items in the SL model (p. 744).

The SL model does not deal with the mirror effect per se, but it does deal with the problem of underlying distributions. The predicted ROC curves (McClelland \& Chappell, 1998, Figure 4, p. 742) for the model capture the essence of both the list strength effect and the list length effect (Balakrishnan \& Ratcliff, 1996). In particular, the predicted curves show mixed-strong $\cong$ purestrong $>$ pure-weak $\cong$ mixed-weak $>$ long lists, so $d^{\prime}($ strong $)>d^{\prime}($ weak $)$ and $d^{\prime}($ mixed $) \cong d^{\prime}$ (pure). Also, the $z$ ROC (normalized) curves were all linear, with slopes of about 0.8 (Ratcliff, McKoon, \& Tindall, 1994). The parameter $\omega_{\mathrm{c}}$ (the criterion at each detector) varied from -4.2 to 0 to generate the $z$ ROC curves, and the simulations used 300 replications to get acceptably small confidence intervals.

However, the authors report that eight parameter values had to be adjusted to obtain these results. Also, the parameters differed somewhat from the parameter values used in previous fits (Table 10; cf. Table 8). Since these parameters were necessary for the 10 distributions
Table 10

The Full EICL Model

$$
\begin{aligned}
& x_{j}=\alpha^{\operatorname{lag} 2}\left\{\alpha^{\operatorname{lag} 1} x_{j-1}+a\left(1-\alpha^{\text {lag1 }} x_{j-1}\right)\right\} \\
& y_{j}=\beta^{\text {lag2 }}\left\{\beta^{\text {lag1 }} y_{j-1}+b\left(1-\beta^{\text {lag1 } 1} y_{j-1}\right)\right\} \\
& z_{j}=\gamma^{\operatorname{lag} 2}\left\{\left(1-\gamma^{\text {lag1 }}\right) z_{j-1}+c\left(1-\alpha^{\text {lag1 }} x_{j-1}\right)\right\}
\end{aligned}
$$

and

$$
s_{j}=x_{j}-y_{j}+z_{j}
$$

Note- - Lag 1 is the lag between the first presentation(s) and the second presentation(s) and lag2 is the lag between the second presentation and the test (P3).

(old- and new-item distributions for the five curves), the model is more descriptive than explanatory in this regard.

Although the SL model can fit the strength-frequency mirror ordering, it cannot be applied to the forced-choice data, because it does not provide any way to generate the new-item null-choice probability values. The new-item detectors are formed at the time of test, and since AN and BN detectors do not exist at that point, probability correct is undefined. Since the BO and AO distributions must arise from the new (i.e., $\mathrm{AN}$ and $\mathrm{BN}$ ) distributions, there does not seem to be any way, at present, to derive predicted values for the six forced-choice inequalities from the model.

REM model. The concept of differentiation has also been used in the REM model (Shiffrin \& Steyvers, 1997), although in a slightly different way. "An episodic image that has been stored during an earlier presentation of the word currently presented [i.e., at retrieval] is termed an s-image (s for same). An episodic image that has been stored during presentation of any other word ... is termed a d-image ( $\mathrm{d}$ for different)" (p. 146). Thus, in REM, differentiation applies to different items, whereas in the SL model, it refers to features within an item. REM uses a parameter $(G)$ for frequency to produce a mirror effect. However, this only defers what requires explanation.

This notion of differentiation seems quite similar to that of the match-filter model (J. A. Anderson, 1973). There are two matched filters (J. A. Anderson, 1973, Figure 1, p. 423), one for old items with a positive drift rate and one for new items with a negative drift rate. This model was applied to data from the Sternberg scanning paradigm (Sternberg, 1966), and the implication is that the new items were somehow inhibited. Although the notion of a negative matched filter (or differentiation) may be quite reasonable for a fixed-set procedure, it is hard to see how it would work with a varied-set procedure. This is especially true when words are used as the study items (Muter \& Murdock, 1977) ${ }^{21}$ and no mechanism to implement this inhibition is proposed.

Source of activation confusions model. The source of activation confusions (SAC) model is another model that has the potential to explain the mirror effect. Origi- 
nally designed for problem solving (Schunn, Reder, Nhouyvanisvong, Richards, \& Stroffolino, 1997), it has been applied to interactions between remember-know judgments and word frequency (Reder et al., 2000). In one respect, this model is very similar to EICL; it bases starting "strength" values on word frequency norms, so in effect, like EICL, it has a lexicon.

Recognition memory decisions are based on activation level, and there are two sources of activation: direct and indirect (my terms). When an item is presented (the continuous recognition memory paradigm was used), the activation level of the presented word is increased above the base level (which comes from the lexicon); this is direct. Also, by virtue of preexisting associations to other items, the activation level of those words is increased as well; this is indirect. Both sources of activation decay with time, but they have different time courses. The direct source decreases according to a power function to an asymptotic value that is determined by the preexperimental base rate (Reder et al., 2000, Equation 1, p. 303). The indirect activation ( $A$ in the model) decreases exponentially with an asymptotic value of $B$ (the direct activation; Equation 2, p. 304).

The model is couched in terms of nodes and links (it could probably be framed in connectionist terms as well), and the links play an important role in determining the amount of activation that spreads. Their strength (denoted $S$ ) decays as well, again by a power function. Actually, it is a sum of power functions (Reder et al., 2000, Equation 4), each a function of the time since that link (representing the co-occurrence of two items) has been activated. To illustrate, the right panel of Figure 5 shows the sum of three power functions, each with the same exponent but different offsets $(0,2$, and $4 \mathrm{sec})$, each starting $500 \mathrm{msec}$ after presentation. The left panel shows the individual power function curves, each with the values of the starting value $c(25)$ and the rate constant $d(.12)$, taken from Table 2. In this figure, the horizontal axis (time) starts with the third presentation, and the other two curves show the strengths of the two prior presentations on the two time scales.

The motivation for the power function forgetting curves was that the power function had previously (J. R.
Anderson \& Schooler, 1991) been applied quite successfully to some early forgetting data (Ebbinghaus, 1913/1964). It is still quite a reasonable choice, since forgetting data often seem to be more like a power function than an exponential function (R. B. Anderson, 2001; Wixted, 2001; Wixted \& Ebbesen, 1997).

As far as word frequency is concerned, SAC predicted (and found in three experiments) more know responses for high- than for low-frequency words for both old and new items. Words were presented up to 10 times, and the model tracked the obtained proportion of remember and know judgments for low-frequency and high-frequency words very well. ${ }^{22}$ (The remember responses were monotonically increasing and approached a limit of 1.0 for both high- and low-frequency words, but the know responses were consistently nonmonotonic. They peaked at about three presentations and, thereafter, decreased quite gradually.) Judging by the error bars, the fits were quite good.

It would have been interesting to see whether SAC could predict the leapfrog effect and, in particular, the forced-choice inequalities. Unfortunately, not enough detail was given in the original article to make this possible. The details of the lexicon were too scanty; it is not clear how many types and tokens there were, what the lags were, or whether any of the lexicon parameters were random variables. The SAC model seems to assume preexisting associations (i.e., links between nodes), but how, when, and what were not specified. Also, there is some ambiguity in the equations; $\Delta A$ (the decrease in current activation) is specified (Reder et al., 2000, Equation 2), but there are no expressions for the absolute value of $A$, which you need for Equation 3 to compute the change in activation of the receiving nodes.

The general thrust of SAC is that co-occurrences (associations) contribute to recognition memory performance, and that is quite different from EICL. To look for a possible rapprochement, I ran a simulation with an EICL-like lexicon with two streams of words, not one. That is, two permutation vectors were set up for 1,000 types (and 7,069 tokens); for each subject, these two vectors were randomized, and with every pair of items, a simple connectionist network was incremented by a

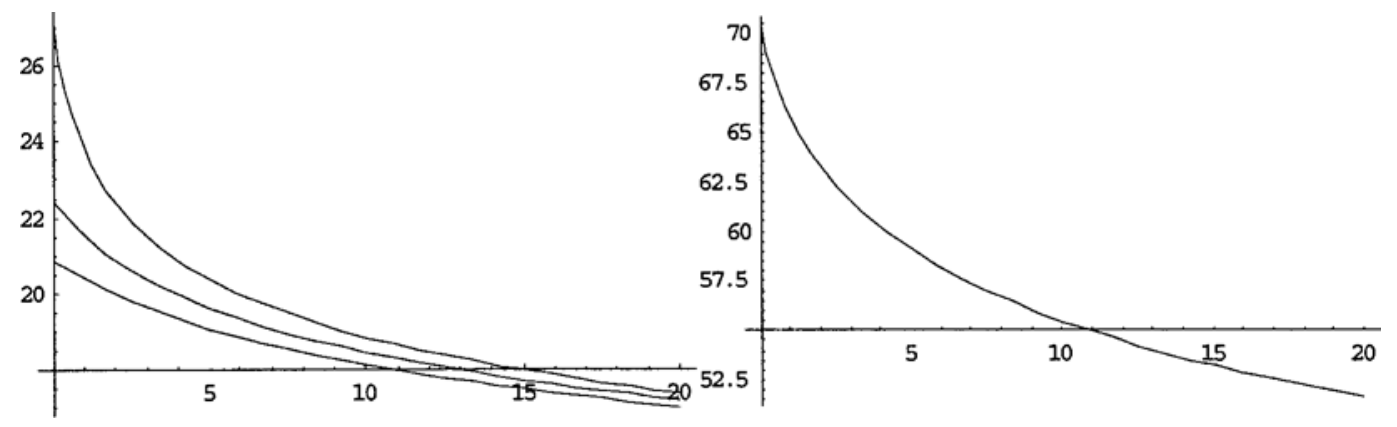

Figure 5. Left panel: Three power functions with the same slope but different onset times. Right panel: Sum of the three for a power function forgetting curve. 
closed-loop algorithm with the Table 4 parameter values. At the end of training this network, or matrix, was a "lexicon" that gave the strengths of the associations between item $i$ and item $j$ for all $i$ s and $j$ s. One can consider "item" strengths to be the diagonal elements $(i=j)$ and the associations the off-diagonal strengths $(i \neq \mathrm{j})$.

Then I computed the forced-choice probability correct values for the high-frequency, or B, items (ranks 130-170), as compared with the low-frequency, or A, items (ranks 830-870), with both item and associative information (diagonals included) or with associative information only (diagonals excluded). However, the program took a very long time to run; 2 subjects required about $24 \mathrm{~h}$. The AN/BN null choice probability correct was .82 and .90 for the 2 subjects, and there was essentially no difference between the two conditions (item and associative or associative only).

Thus, this approach clearly does not give the right results for the AN/BN forced choice, and this is only the first step. However, it is different in many ways from SAC. One could argue that it captures the direct strength, but not the indirect strength, aspect of SAC, but even this is debatable. In any case, given these uncertainties, there seemed to be no point in running further simulations.

Consequently, the main point here is that whether $\mathrm{SAC}$ can explain the mirror effect (and the forced-choice probabilities, in particular) remains to be seen. However, there is a possible problem for the general framework of SAC that should not pass without notice. Decay is time based for both direct and indirect activation; this would seem to predict that, in recognition memory, the faster the presentation rate, the more accurate recognition should be, and this is clearly wrong. There is some compelling data showing improvement in recognition memory from 25 to $500 \mathrm{msec}$ (Loftus, 1974), and in some of our own experiments on recognition we found similar effects over longer intervals (Murdock \& Anderson, 1975). This problem might be solved by making the learning rate constants time dependent. ${ }^{23}$

The target episode cue object (TECO) model. There is one other model I know of that can explain not only the forced-choice regularities, but also the other mirror regularities as well, and that is the TECO model (Sikström, 1996, 2000). TECO is a connectionist model that has separate item and context components that are associated by an outer-product operation. For the mirror effect, the model assumes that the mean strengths of high- and low-frequency items are the same but the variances differ. In particular, the variance is greater for high-frequency items than for low-frequency items. Then, if the criterion (strength-based, not Bayesian) is set anywhere between the new- and the old-item means and the decision is based on the cumulative (cf. probability) distributions, the mirror effect falls out naturally.

The justification for the key assumption (unequal variances) is that high-frequency items are experienced in more different contexts than are low-frequency items. Although this is reasonable, it is not clear why the means for new A and B items should be the same, and no mechanism to implement the distributional assumptions is suggested. Thus, the same criticism (that the model is assuming what needs to be explained) would seem to apply here too. If it could be shown how the new-item distributions are generated and then show how the same rules and parameter values can generate the old-item distributions, this would be a very powerful and impressive model indeed. Perhaps this is a job for the future.

\section{Interactions}

Recent experiments have extended our knowledge about the mirror effect by finding interactions between word frequency and other variables. One example is the SAC model, which led to the prediction of differential effects on remember and know judgments. Another example (Joordens \& Hockley, 2000) dealt with recollection and familiarity, but these are two pairs of names that seem to have the same referent as remember and know. The thrust of this second example is that there are manipulations (e.g., word frequency), during both the study phase and the test phase, that affect the hit rate part of the mirror effect but do not affect the false alarm rate part of the mirror effect.

To illustrate the differences between remember/recollect and know/familiarity, the following instructions were used in Joordens and Hockley's (2000) study. Remember includes recollection, conscious awareness of some aspect of what happened, appearance, what you were thinking of, or something else you noticed. Know is the absence of all of these (Gardiner \& Java, 1990).

Another possible interpretation of such interactions is in terms of a "single"-process view (Donaldson, 1996). By this view, recollection or remember judgments are associated with high-confidence yes responses, but know or familiarity judgments are associated with lower confidence yes responses. This "single" process (I use quotes because signal detection always involves two processes, memory and decision) can provide a simple explanation of such interactions. If you think of the four distributions aligned as in Figure 1 with the no-yes criterion bisecting the center and another criterion above that for remember/recollect, it is reasonable to suggest that these manipulations (whatever their nature) shift the AO distribution down, relative to the BO distribution, but do not affect the new-item distribution or the yes-no criterion. This provides a very simple strength-based explanation for their interactions. However, again, this is describing what needs to be explained, and it would have to be spelled out in more detail to constitute a satisfactory explanation.

In the discussion, the authors argue that a "single-factor perspective" cannot account for their data. What they refer to, in particular, are rescaling theories ${ }^{24}$-in particular, attention-likelihood theory. The reason attentionlikelihood theory cannot account for the data is that it uses the log-likelihood ratio, and this ratio (ordinates of old items to new items) ties both distributions together in 
the decision stage. In contrast, the SL model uses log odds, not log-likelihood ratio, so the SL model is not subject to this criticism.

Although a dual-process view may provide an easy interpretation of such interactions, there are other results that are not so easily explained. Two such results are the a posteriori probability results and the associated latencies of correct responses from studies of recognition memory in which a confidence judgment procedure was used. The a posteriori probability functions (probability of an old response, given a particular confidence judgment) are monotonic from --- to +++ , covering the range from .05 to .95 . For the latency/confidence results, mediumconfidence judgments are about $250 \mathrm{msec}$ longer than high-confidence responses, and low-confidence responses are about $250 \mathrm{msec}$ longer than medium-confidence judgments, for both old and new responses (Murdock, 1980). These results would seem to argue strongly for a continuous strength view, as in EICL, and they provide a challenge for dual-process advocates to explain. ${ }^{25}$

The SAC model takes a somewhat different view of the dual processes. It assumes that remember (recollection) judgments involve associative information, as well as item information, whereas know (familiarity) judgments lack the associative component. The instructions to subjects emphasize this distinction. This distinction is similar in spirit to TODAM2, which distinguishes between item information and associative information. TODAM2 uses this distinction to explain some interactions between item information and associative information (Murdock, Smith, \& Bai, 2001). Both item strength and associative strength are continuous, so in principle, neither the a posteriori probability/latency results nor the remember/know dissociations are inconsistent with TODAM2. However, EICL must be incorporated into TODAM2 if TODAM2 is to explain the mirror and the spacing effects. ${ }^{26}$

Class strengthening. Another type of interaction comes from studies of class strengthening. As in Joordens and Hockley's (2000) study, if a manipulation such as differential strengthening (say, by repetition) is used for two classes of items (say, high- and low-frequency words) and subjects use a likelihood ratio principle for their decision (as in ALT and REM), the strengthening manipulation should not differentially affect hits and false alarms if the manipulation is within, rather than between, lists. In fact, it does (Morrell, Gaitan, \& Wixted, 2002; Stretch \& Wixted, 1998), and in these class-strengthening studies, one finds the same general effect as in Joordens and Hockley's study-namely, an effect on the hit rate, but not on the false alarm rate. Also, Reder et al. (2000) got the same pattern of results.

One possibility is that subjects use different criteria for the two classes of words (i.e., high frequency and low frequency). The authors (i.e., Stretch \& Wixted, 1998, and Wixted, 2001) feel that this is unlikely, since it would necessitate an item-by-item adjustment of the criteria over the course of testing. Another possibility is that, in fact, subjects do not use a likelihood ratio principle as the basis for decision. Likelihood ratios are part of Bayesian inference, and there is clear evidence that subjects do not revise subjective probabilities in accordance with Bayes's rule (Coombs, Dawes, \& Tversky, 1970, pp. 145-147). More direct evidence comes from a study that tested whether subjects used a likelihood ratio principle in a recognition memory task (Creelman \& Donaldson, 1968). They found that the "a priori probability did not affect measured discrimination. The data suggest that a decision criterion was set to match response proportions to the a priori stimulus probability" (p. 514).

Although a likelihood ratio principle for decision is attractive and Bayesian inference seems to be becoming more popular in memory-and-decision models, the evidence, such as it is (Dodhia, 2000), does not seem too supportive. The alternative, of course, is that subjects use strength, rather than likelihood, for their decisions, and this is consistent with EICL, as well as with some global-matching models (e.g., SAM, TODAM, CHARM, and MINERVA). 27

\section{Spacing Effect}

Consideration of the spacing effect can be much briefer than that of the mirror effect, because there has been relatively little theoretical work on this issue in the last 20 years or so. There are several excellent detailed reviews of the early work on the spacing effect and its theoretical significance (Crowder, 1976; Hintzman, 1974). In the latter review, the five possible explanations discussed were (in increasing probability of being correct) rehearsal, encoding variability, consolidation, attention, and habituation. The former review suggested that encoding variability could be a more promising approach if variability in context were the key factor. Early models for the spacing effect (Bower, 1972; Glenberg, 1976), based on stimulus fluctuation theory (Estes, 1955), formalized these ideas. The approach taken here extends these ideas, with some additional explanatory notions.

How does EICL compare with other models of the spacing effect? As has been mentioned, in one review of the spacing effect (Hintzman, 1974), it was suggested that the most likely candidates for an explanation of the spacing effect were context-based encoding variability and habituation. Several early models (Bower, 1972; Glenberg, 1976) also stressed the importance of context. Hopefully, it is clear that EICL is quite compatible with the context-based interpretation, even though the mechanisms are not detailed in the same way. Also, EICL is quite compatible with a habituation notion. Recent models of habituation (Sikström, 2003; Staddon \& Higa, 1996) assign an important role to inhibitory processes, and the same is true in EICL.

Optimality model. There is one model that can fit the spacing effect data at a quantitative level; in fact, it can do a spectacularly good job. At least by a pattern match 
criterion (the absolute values are slightly off), the fits to some complex spacing effect data (Glenberg, 1976) could not be better. This model is the optimality model (J. R. Anderson \& Schooler, 1991; our term), which also provides the underpinnings of the SAC model (see Equation 1 in Reder et al., 2000). The following are the key assumptions of the optimality model: With multiple presentations of an item, " 1 . The strengths from individual presentations sum to produce a total strength; 2 . Strengths of individual presentations decay as a power function of the time; 3 . The exponents of the power function for decay of each presentation decreases as a function of time since previous presentations" (J. R. Anderson \& Schooler, 1991, p. 406).

Consider the second and third assumptions: The individual strengths decay as a power function, and their decay rates (exponents) also decay as a power function. In particular, the decay rate for the memory trace of the $i$ th presentation is the maximum of the decay rate of the initial presentation and a function $(b)$ of the reciprocal $\left(d_{i}\right)$ of the spacing between the previous and the current presentation $\left(t_{i}-t_{i-1}\right)$; all this comes from Equation 11 (J. R. Anderson \& Schooler, 1991, p. 407). Thus, not only are power function decay rates assumed, but also the decay rates themselves for each particular presentation are a rather complex function of the spacing. There is more to the model than this, ${ }^{28}$ but for our purposes, these are the critical points.

This optimality model would also seem to be vulnerable to the criticism that it assumes what needs to be explained. Power curve forgetting is assumed because this best fits the data, but recent developments (R. B. Anderson, 2001; Kahana \& Adler, 2003) have shown that power curve forgetting functions can be derived by assuming variability in the parameters of other underlying forgetting functions. ${ }^{29}$ The deeper assumption (that the decay rates of the individual memory traces themselves decay as a function of spacing) is exactly the sort of "explanation" that EICL is trying to avoid. Although the optimality model does provide a detailed model for the data, as the authors themselves acknowledge, this just moves the problem from explaining the spacing effect to explaining why the decay rates themselves decay by a power function.

Thus, you can explain the spacing effect by assuming that the decay rates of the individual memory traces themselves decay as a function of spacing, but then you have to explain why the decay rates (exponents of assumed power functions) decay as a power function of lag. Also, it is far from clear what sort of a mechanism or process would function like this. It is one thing to say that the strength of an item decays as a power function of time, but quite another to say that the decay rate itself is a function of the number of presentations. How does the memory system remember what the decay rate for each presentation is? There are other multiplex (localized) models (e.g., MINERVA, SAM), but none of them makes an assumption like this. However, as J. R. Ander- son and Schooler (1991) say, "Although deeper mechanistic explanations would be nice, we think it is an accomplishment to finally have mathematical functions that can capture the effects of practice and delay" (p. 407). It is hard to disagree with this sentiment, but now, 10 years latter, it is not the only one.

\section{SUMMARY AND CONCLUSION}

EICL consists of three stochastic difference (or update) equations, one each for excitation, inhibition, and context (Table 10). All are closed-loop equations, so the increment is always proportional to the amount remaining. Each has its own learning rate constant and forgetting parameter, and the increment takes into account what had been forgotten since the previous presentation. This requires six parameters (not necessarily all different), one each for the learning rate constants and one each for the forgetting parameters. All the parameters are random variables, random samples from truncated normal distributions, each with its own mean and coefficient of variation, which determine the standard deviation. This may seem like a lot of parameters, but it is comparable to the number of parameters required in SAM, REM, SL, SAC, and the network model of the hippocampus.

SL and SAC (for links) assume exponential forgetting, but the optimality model assumes power function forgetting. EICL assumes exponential forgetting with variability, which results in an approximation to power function curves. Like SAC, EICL includes a lexicon, so starting values are derived, not assumed. However, strength (activation) in SAC is based on pairwise connections (associative information), whereas in EICL, strength is based on item information-more specifically, the algebraic sum of the three components. Context does not affect the lexicon in EICL or in SAC, but (by one interpretation of episodic nodes) it does affect the study phase results. Assumptions about context determine the starting values for strength in TECO, but context is not included in SL.

What exactly are the main contributions of EICL? I think they are four in number. First, EICL specifies the mechanisms that can generate most of the right patterns of data for two very puzzling phenomena, the mirror effect and the spacing effect, and can do so with the same parameter values. All the parameter values were exactly the same for both effects.

Second, none of the parameter values varied with the independent variables (word frequency for the mirror effect, lag for the spacing effect). What produced the mirror effect was the difference in number of lexicon repetitions (word frequency), which then determined lag, which then (by the update equations) determined strength. What produced the spacing results was the joint action of $\operatorname{lag} 1$ and $\operatorname{lag} 2$, and these in turn determined the strengths according to the update equations. Any model that uses different parameter values for high- and low-frequency 
words is not really explaining the effect, because one must then ask why the parameter values change the way they do. One can make the same argument about the spacing effect.

Third, the starting strength distributions were derived from the toy lexicon; they were not assumed. As I showed in the initial example, it is very easy to generate a mirror ordering if one assumes the starting values, so this is a description of the data, not an explanation. I worry even more when the starting distributions are assumed. In the course of this investigation, I have found several distributions with a variety of characteristics (means, shapes, and variances) that can produce reasonable forcedchoice inequalities. ${ }^{30}$ The problem, however, is to show how they came into being. The same learning rules with the same parameter values should be used to generate a lexicon and increment the strength values in the study phase.

Fourth and finally, EICL incorporates both individual differences and a (very crude) semantic memory into an episodic memory model. No one will deny that there are individual differences, both between subjects and between items, and that these are included in the model. Every parameter is treated as a random variable.

Although EICL cannot describe the fine-grained detail of the data as well as some other models, it does generate the right patterns of data for the mirror effect and the spacing effect. The predictions do not match the data in all cases, but this could be because it is incomplete, rather than wrong. The roots of these ideas go back at least as far as the mid-1950s (Bush \& Mosteller, 1955; Estes, 1955), but EICL tries to combine them in the right way. However, the model needs to be extended to account for other recognition memory effects, and perhaps this can be accomplished by embedding it in a globalmatching model.

\section{REFERENCES}

Adams, J. A. (1967). Human memory. New York: McGraw-Hill.

Anderson, J. A. (1973). A theory for the recognition of items from short memorized lists. Psychological Review, 80, 417-438.

Anderson, J. R., \& Schooler, L. J. (1991). Reflections of the environment in memory. Psychological Science, 2, 396-408.

Anderson, R. B. (2001). The power law as an emergent property. Memory \& Cognition, 29, 1061-1068.

BALAKRishnan, J. D., \& RATClifF, R. (1996). Testing models of decision making using confidence ratings in classifications. Journal of Experimental Psychology: Human Perception \& Performance, 22, 615-633.

Bogartz, W. (1968). A small error in Thurstone's length-difficulty function. Psychological Review, 75, 442-445.

Bower, G. H. (1971). Adaptation-level coding of stimuli and serial position effects. In M. H. Appley (Ed.), Adaptation-level theory (pp. 175201). New York: Academic Press.

BowER, G. H. (1972). Stimulus-sampling theory of encoding variability. In A. W. Melton \& E. Martin (Eds.), Coding processes in human memory (pp. 85-123). Washington, DC: Winston.

Bush, R. R., \& Mosteller, F. (1955). Stochastic models for learning. New York: Wiley.

Coombs, C. H., Dawes, R. M., \& Tversky, A. (1970). Mathematical psychology: An elementary introduction. Englewood Cliffs, NJ: Prentice-Hall.
Creelman, C. D., \& Donaldson, W. (1968). ROC curves for discrimination of linear extent. Journal of Experimental Psychology, 77, 514-516.

Crowder, R. G. (1976). Principles of learning and memory. Hillsdale, NJ: Erlbaum.

Dennis, S., \& Humphreys, M. S. (2001). A context noise model of episodic word recognition. Psychological Review, 108, 452-478.

DoDHIA, R. M. (2000). Subjective judgments of likelihood evidence. Unpublished doctoral dissertation, Columbia University.

DonaldSon, W. (1996). The role of decision processes in remembering and knowing. Memory \& Cognition, 24, 523-533.

Dragoi, V., \& Staddon, J. E. R. (1999). The dynamics of operant conditioning. Psychological Review, 106, 20-61.

EbBinghaus, H. (1964). Memory: A contribution to experimental psychology. New York: Teachers College, Columbia University. (Reprinted by Dover; originally published 1913)

ESTES, W. K. (1955). Statistical theory of spontaneous recovery and regression. Psychological Review, 62, 145-154.

ESTES, W. K. (1972). Coding processes in human memory. Washington, DC: Winston.

GARDINER, J. M., \& JAVA, R. I. (1990). Recollective experience in word and nonword recognition. Memory \& Cognition, 18, 23-30.

Gillund, G., \& \& Shiffrin, R. M. (1984). A retrieval model for both recognition and recall. Psychological Review, 91, 1-67.

Glanzer, M., \& ADAMs, J. K. (1985). The mirror effect in recognition memory. Memory \& Cognition, 13, 8-20.

Glanzer, M., Adams, J. K., \& Iverson, G. [J.] (1991). Forgetting and the mirror effect in recognition memory: Concentering of underlying distributions. Journal of Experimental Psychology: Learning, Memory, \& Cognition, 17, 81-93.

Glanzer, M., Adams, J. K., Iverson, G. J., \& KIm, K. (1993). The regularities of recognition memory. Psychological Review, 100, 546567.

Glanzer, M., Kim, K., Hilford, A., \& Adams, J. K. (1999). Slope of the receiver-operating characteristic in recognition memory. Journal of Experimental Psychology: Learning, Memory, \& Cognition, 25, 500-513.

GLenberg, A. M. (1976). Monotonic and nonmonotonic lag effects in paired-associate and recognition memory paradigms. Journal of Verbal Learning \& Verbal Behavior, 15, 1-16.

Green, D. M., \& Moses, F. L. (1966). On the equivalence of two recognition measures of short-term memory. Psychological Bulletin, 66, 228-234.

Greene, R. L. (1992). Human memory: Paradigms and paradoxes. Hillsdale, NJ: Erlbaum.

GrossBerg, S. (1978). Do all neural models really look alike? A comment on Anderson, Silverstein, Ritz, and Jones. Psychological Review, 85, 592-596.

Groves, P. M., \& Thompson, R. F. (1970). Habituation: A dual-process theory. Psychological Review, 77, 419-450.

Haberlandt, K. (1999). Human memory. Boston: Allyn \& Bacon. Hasselmo, M. E., \& Wyble, B. P. (1997). Free recall and recognition in a network model of the hippocampus: Simulating effects of scopolamine on human memory function. Behavioural Brain Research, 89 , $1-34$.

Hintzman, D. L. (1969). Recognition time: Effects of recency, frequency, and the spacing of repetitions. Journal of Experimental Psychology, 79, 192-194.

HintzMan, D. L. (1974). Theoretical implications of the spacing effect. In R. L. Solso (Ed.), Theories in cognitive psychology (pp. 7799). Potomac, MD: Erlbaum.

Hintzman, D. L. (1988). Judgments of frequency and recognition memory in a multiple-trace memory model. Psychological Review, 95, 528-551.

Hintzman, D. L. (1994). On explaining the mirror effect. Journal of Experimental Psychology: Learning, Memory, \& Cognition, 20, 201205.

Hintzman, D. L., Caulton, D. A., \& Curran, T. (1994). Retrieval constraints and the mirror effect. Journal of Experimental Psychology: Learning, Memory, \& Cognition, 20, 275-289.

Horn, D., \& UsHer, M. (1990). Excitatory-inhibitory networks with 
dynamical thresholds. International Journal of Neural Systems, $\mathbf{1}$, 249-257.

Hurvich, L. M., \& JAMESON, D. (1974). Opponent process as a model of neural organization. American Psychologist, 29, 88-102.

Joordens, S., \& HockLey, W. E. (2000). Recollection and familiarity through the looking glass: When old does not mirror new. Journal of Experimental Psychology: Learning, Memory, \& Cognition, 26, 1534-1555.

KahANA, M. J., \& ADler, M. (2003). Note on the power law of forgetting. Manuscript submitted for publication.

KANDEL, E. R. (2001). The molecular biology of memory storage: A dialogue between genes and synapses. Science, 294, 1030-1038.

Kelley, R., \& WiXted, J. T. (2001). On the nature of associative information in recognition memory. Journal of Experimental Psychology: Learning, Memory, \& Cognition, 27, 701-722.

Kolb, B., \& Whishaw, I. Q. (1996). Fundamentals of neuropsychology (4th ed.). New York: Freeman.

Kwantes, P. J., \& Mewhort, D. J. K. (1999). Modeling lexical decision and word naming as a retrieval process. Canadian Journal of Experimental Psychology, 53, 306-315.

LEWANDOWSKY, S. (1999). Redintegration and response suppression in serial recall: A dynamic network model. International Journal of Psychology, 34, 434-446.

LEWANDOWSKY, S., \& MURDock, B. B. (1989). A distributed model for associative learning. In D. Vickers \& P. L. Smith (Eds.), Human information processing: Measures, mechanisms, and models (pp. 381394). Amsterdam: North-Holland.

Li, S.-C., Lewandowsky, S., \& DeBrunner, V. E. (1996). Using parameter sensitivity and interdependence to predict model scope and falsifiability. Journal of Experimental Psychology: General, 125, 360-369.

Li, S.-C., Lindenberger, U., \& Frensch, P. A. (2000). Unifying cognitive aging: From neuromodulation to representation to cognition. Neurocomputing, 32-33, 879-890.

LofTUs, G. R. (1974). Acquisition of information from rapidly presented verbal and nonverbal stimuli. Memory \& Cognition, 2, 545-548.

Maddox, W. T., \& Estes, W. K. (1997). Direct and indirect stimulusfrequency effects in recognition. Journal of Experimental Psychology: Learning, Memory, \& Cognition, 23, 539-559.

Malmberg, K. J., \& Murnane, K. (2002). List composition and the word-frequency effect for recognition memory. Journal of Experimental Psychology: Learning, Memory, \& Cognition, 28, 616-630.

McClelland, J. L., \& Chappell, M. (1998). Familiarity breeds differentiation: A subjective-likelihood approach to the effects of experience in recognition memory. Psychological Review, 105, 734-760.

McDowd, J. M., \& Murdock, B. B. (1986). Mathematical models of memory and the problem of stimulus variation: A comparison of MINERVA2 and TODAM. Acta Psychologica, 62, 177-188.

Morrell, H. E. R., Gaitan, S., \& Wixted, J. T. (2002). On the nature of the decision axis in signal-detection-based models of recognition memory. Journal of Experimental Psychology: Learning, Memory, \& Cognition, 28, 1095-1110.

Murdock, B. B. (1967). Recent developments in short-term memory. British Journal of Psychology, 58, 421-433.

Murdock, B. B. (1980). Short-term recognition memory. In R. S. Nickerson (Ed.), Attention and performance VIII (pp. 497-519). Hillsdale, NJ: Erlbaum.

Murdock, B. B. (1997). Context and mediators in a theory of distributed associative memory (TODAM2). Psychological Review, 104, 839-862.

Murdock, B. B. (1998). The mirror effect and attention-likelihood theory: A reflective analysis. Journal of Experimental Psychology: Learning, Memory, \& Cognition, 24, 524-534.

Murdock, B. B., \& Anderson, R. E. (1975). Encoding storage, and retrieval of item information. In R. L. Solso (Ed.), Information processing and cognition: The Loyola symposium (pp. 145-194). Hillsdale, NJ: Erlbaum.

Murdock, B. [B.], \& LAMon, M. (1988). The replacement effect: Repeating some items while replacing others. Memory \& Cognition, 16, 91-101.

Murdock, B. B., Smith, D., \& BAI, J. (2001). Judgments of frequency and recency in a distributed memory model. Journal of Mathematical Psychology, 45, 564-602.

Muter, P., \& Murdock, B. B., JR. (1977). A search for scanning residue in recognition memory. Bulletin of the Psychonomic Society, 10, 66-68.

Peterson, L. R., Wampler, R., Kirkpatrick, M., \& Saltzman, D. (1963). Effect of spacing presentations on retention of a paired associate over short intervals. Journal of Experimental Psychology, 66, 206-209.

RadVAnsky, G. A. (1999). Memory retrieval and suppression: The inhibition of situation models. Journal of Experimental Psychology: General, 128, 563-579.

Ratcliff, R., McKoon, G., \& Tindall, M. (1994). Empirical generality of data from receiver-operating characteristic functions and implications for global memory models. Journal of Experimental Psychology: Learning, Memory, \& Cognition, 20, 763-785.

Ratcliff, R., Sheu, C.-F., \& Gronlund, S. D. (1992). Testing global memory models using ROC slopes. Psychological Review, 99, 518535.

Reder, L. M., Nhouyvanisvong, A., Schunn, C. D., Ayers, M. S., Angstadt, P., \& Hiraki, K. (2000). A mechanistic account of the mirror effect for word frequency: A computational model of rememberknow judgments in a continuous recognition paradigm. Journal of Experimental Psychology: Learning, Memory, \& Cognition, 26, 294320.

Schunn, C. D., Reder, L. M., Nhouyvanisvong, A., Richards, D. R., \& Stroffolino, P. J. (1997). To calculate or not to calculate: A source activation confusion model of problem familiarity's role in stategy selection. Journal of Experimental Psychology: Learning, Memory, \& Cognition, 23, 3-29.

Shepard, R. N., \& Chang, J. (1963). Forced-choice tests of recognition memory under steady-state conditions. Journal of Verbal Learning \& Verbal Behavior, 2, 93-101.

Shiffrin, R. M., \& Steyvers, M. (1997). A model for recognition memory: REM-retrieving effectively from memory. Psychonomic Bulletin \& Review, 4, 145-166.

SIKsтröm, S. (1996). The TECO connectionist theory of recognition failure. European Journal of Cognitive Psychology, 8, 341-380.

SiKSTRöm, S. (2000). The TECO theory and lawful dependency in successive episodic tests. Quarterly Journal of Experimental Psychology, 53A, 693-728.

SIKSTRÖM, S. (2001). The variance theory of the mirror effect in recognition memory. Psychonomic Bulletin \& Review, 8, 408-438.

SiKSTRÖM, S. (2003). The habituation model for episodic memory. Manuscript submitted for publication.

SMITH, D. G. (2003). Theories of confidence-judgment for recognition of items, associations, and source. Manuscript submitted for publication.

Smith, D. G., \& Niewiadomski, M. W. (2001, November). Frequency effects in recognition memory: Evidence for a multiprocess detection model. Paper presented at the 42nd Annual Meeting of the Psychonomic Society, Orlando, FL.

Solomon, R. L. (1980). The opponent-process theory of acquired motivation. American Psychologist, 35, 691-712.

Spence, K. W. (1937). The differential response in animals to stimuli varying within a single dimension. Psychological Review, 44, 430-444.

StADDON, J. E. R. (1993). On rate-sensitive habituation. Adaptive Behavior, 1, 421-436.

Staddon, J. E. R., \& Higa, J. J. (1996). Multiple time scales in simple habituation. Psychological Review, 103, 720-733.

Sternberg, S. (1966). High-speed scanning in human memory. Science, 153, 652-654.

Stretch, V., \& Wixted, J. T. (1998). On the difference between strength-based and frequency-based mirror effects in recognition memory. Journal of Experimental Psychology: Learning, Memory, \& Cognition, 24, 1379-1396.

Thurstone, L. L. (1930). The learning function. Journal of General Psychology, 3, 469-493.

Townsend, J. T., \& Busemeyer, J. R. (1978). Approach-avoidance: Return to dynamic decision behavior. In C. Izawa (Ed.), Current issues in cognitive psychology: The Tulane Floweree Symposium on Cognition (pp. 107-133). Hillsdale, NJ: Erlbaum. 
Usher, W., \& MCClelland, J. L. (2001). The time course of perceptual choice: The leaky, competing accumulator model. Psychological Review, 108, 550-592.

WiXted, J. [T.] (2001, November). In defense of the power law of forgetting. Paper presented at the 42nd Annual Meeting of the Psychonomic Society, Orlando, FL.

Wixted, J. T., \& EbBesen, E. B. (1997). Genuine power curves in forgetting: A quantitative analysis of individual subject forgetting functions. Memory \& Cognition, 25, 731-739.

Yonelinas, A. P. (1999). The contribution of recollection and familiarity to recognition and source-memory judgments: A formal dualprocess model and an analysis of receiver operating characteristics. Journal of Experimental Psychology: Learning, Memory, and Cognition, 25, 1415-1434.

Yonelinas, A. P., Hockley, W. E., \& Murdock, B. B. (1992). Tests of the list-strength effect in recognition memory. Journal of Experimental Psychology: Learning, Memory, \& Cognition, 18, 345-355.

ZIPF, G. K. (1945). The repetition of words, time-perspective, and semantic balance. Journal of General Psychology, 32, 127-148.

\section{NOTES}

1. In a forced-choice procedure, the subject is presented with two (or more) alternatives and is asked to pick the stronger (or older, more recent, or whatever). It has long been used in studies of recognition memory (Green \& Moses, 1966).

2. Null choices are sometimes regarded as "artificial." How can subjects make a rational choice between two items in the same category? But where would psychophysics be today without the study of threshold differences?

3. Figure 1 , as drawn, would imply that $\mathrm{AN} / \mathrm{BN} \cong \mathrm{BN} / \mathrm{BO} \cong \mathrm{BO} / \mathrm{AO}$, but Figure 1 is really only heuristic, not a formal model.

4. However, the closed-loop algorithm might have trouble explaining data on judgments of frequency (Hintzman, 1988). Also, it did not fit some recognition memory data, using a replacement procedure (Murdock \& Lamon, 1988), but context was not included.

5. In another terminology, these equations are the closed-loop equivalent of leaky integrator models. These models have been applied with considerable success to such problems as operant conditioning and perceptual choice (Dragoi \& Staddon, 1999; Usher \& McClelland, 2001).

6 . The usual definition of a lexicon seems to be that of a dictionarythat is, a list of words and their meanings. Here, all I mean by lexicon is a list of items that can be recognized as words; no meanings are included-in other terms, item information, not associative information.

7. All parameter values were restricted so as to lie in the interval $(0$, 1), so in effect, the parameters were sampled from truncated normal distributions. The degree of truncation varied with the parameters.

8. The same was true in using TODAM2 to fit some judgment-offamiliarity and judgment-of-recognition data (Murdock, Smith, \& Bai, 2001), and I was able to use the same parameter value (.97) for context drift. However, to avoid confusion, here it is called $\gamma$, rather than $\rho$.

9. This value was arbitrary. The exact value did not seem to make much difference as long as it was not too large.

10. In particular, $\alpha$ and $\beta$ were varied somewhat haphazardly in the range of .99995-.99999, the scale factors for lag variability from 0.8 to 1.2 , and the learning rate constants for inhibition $(b)$ and context $(c)$ from .008 to .010 and from 0.40 to 0.50 .

11. At the suggestion of one of the reviewers, several update equations that did not work are given in the Appendix.

12. Variances are generally inferred from $z$ ROC curves (parametric plots of $z$-transformed hit rates as a function of $z$-transformed false alarm rates), but these curves assume underlying normal distributions. To my knowledge, it has not been shown that the same conclusion follows when the underlying distributions are not normal.

13. There was a small error in the derivation (Bogartz, 1968).

14. Not only is the rate of habituation a function of the presentation rate of the stimulus, but the recovery rate is as well.

15. I thank John Antrobus for calling this article to my attention.

16. "Generally" because of the sigmoidal mapping function, rather than a step function.

17. We may be latecomers in using excitatory-inhibitory notions for memory models. Sophisticated models of this sort have existed in the artificial neural network field for some time (e.g., Horn \& Usher, 1990).

18. Use of the log-likelihood ratio has been questioned (Hintzman, Caulton, \& Curran, 1994), but perhaps it is not completely unreasonable (Murdock, 1998).

19. An empirical problem for ALT is claimed to be the effect of list composition (proportion of high- and low-frequency items; Malmberg \& Murnane, 2002).

20. The likelihood is the product of probabilities and, from a statisticalinference point of view, serves as the best estimate of the population values - that is, the features in the item vectors.

21. It is interesting to note that in this study, which used words drawn from the Toronto word pool, the slopes of the scanning function were only about $12 \mathrm{msec} /$ item. This study may be found on the first author's Web page, which is http://psych.utoronto.ca/ muter/.

22. One interpretation of remember and know in terms of the theory of signal detection is that remember judgments are simply higher [sic] confidence responses than know responses (Donaldson, 1996). However, this was not the SAC definition; there is more on this point in the Interactions section.

23. This is how TODAM deals with presentation-rate effects (Murdock \& Lamon, 1988).

24. Rescaling may not be a good term. There is a function (or perhaps several functions) mapping one variable (or set of variables) into another. What is important is the nature of the mapping function(s), not the fact that some mapping is assumed.

25. Hybrid models (Smith, 2003; Yonelinas, 1999) would seem not to be bothered by these criticisms. However, a potential problem for onedimensional models is that, with a pure-lists design, the mirror effect changes; hit rate differences between high-frequency and low-frequency words disappear, and the false alarm rate pattern changes (Smith \& Niewiadomski, 2001).

26. Additional interactions of item and associative information (Kelley \& Wixted, 2001) may raise problems for this view, but further discussion of these issues would take us too far afield.

27. The cross-comparison data is not problematic for the SL model, because it uses log odds, not the log-likelihoodratio, as the basis for decision, so there is no necessity that responses to new items track responses to old items.

28. This includes Bayesian inference, which is why I am calling it an optimality model; the authors do not suggest a name.

29. A still more recent result suggests that, by extrapolation, power function decay curves do provide a better fit to data than do exponential decay functions (Wixted, 2001), but this does not negate the theoretical point.

30 . One situation is that if $\operatorname{Var}[\mathrm{BN}] \cong \operatorname{Var}[\mathrm{BO}]<\operatorname{Var}[\mathrm{AN}] \cong$ $\operatorname{Var}[\mathrm{BN}],(\mathrm{E}[\mathrm{AO}]-\mathrm{E}[\mathrm{AN}])>\mathrm{E}[\mathrm{BO}]-\mathrm{E}[\mathrm{BN}]$ and the distributions are symmetrically placed about the midpoint, then, using a numerical integration package, one ge ts nice forced-choice values for normal, binomial, and uniform distributions. The same is true if the expectations (E) have the mirror ordering and the A item variances are greater [sic] than the B item variances. 


\section{APPENDIX}

There are update equations for context that will not give the right results, for both the mirror effect and the spacing effect. The following equations give the right results for the mirror effect, but not for the spacing effect:

$$
\begin{aligned}
& x_{j}=\alpha^{\operatorname{lag}} x_{j-1}+a\left(1-\alpha^{\operatorname{lag}} x_{j-1}\right), \\
& y_{j}=\beta^{\operatorname{lag}} y_{j-1}+b\left(1-\beta^{\operatorname{lag}} y_{j-1}\right),
\end{aligned}
$$

and

$$
z_{j}=\rho^{\operatorname{lag}} z_{j-1}+c\left(1-\alpha^{\operatorname{lag}} x_{j-1}\right) .
$$

The following equations give the right results for the spacing effect, but not for the mirror effect:

$$
\begin{aligned}
& x_{j}=\alpha^{\operatorname{lag}} x_{j-1}+a\left(1-\alpha^{\operatorname{lag}} x_{j-1}\right), \\
& y_{j}=\beta^{\operatorname{lag}} y_{j-1}+b\left(1-\beta^{\operatorname{lag}} y_{j-1}\right),
\end{aligned}
$$

and

$$
z_{j}=\rho^{\operatorname{lag}} z_{j-1}+c\left(1-\rho^{\mathrm{lag}}\right) x_{j-1} .
$$

As an example, consider the mirror effect that one gets with the first set of update equations with $c=0.4$ $\pm .05,500$ subjects, and the other parameters being essentially the same as those in Table 4 . The mirror ordering was $.379, .494, .576$, and .633 , and the forced-choice probability correct values were $.698, .619, .667$, $.810, .787$, and .898 . One gets a nice leapfrog effect: for the mirror ordering, $(\mathrm{AO}-\mathrm{AN})>>(\mathrm{BO}-\mathrm{BN})$, and for the forced-choice probability correct values, $\mathrm{AO} / \mathrm{AN}>>\mathrm{BO} / \mathrm{BN}$ (.898 vs. .667). Even the distributions are quite nice (Figure A1). However, with this context equation, one will not get any leapfrog effect for spacing, and this is what makes the joint fits to the mirror effect and the spacing effect more difficult than fits to either one alone.

Another way to get a mirror effect is to assume upper and lower strength boundaries so that items whose strength is above the upper boundary are not incremented when they occur. That is, they are ignored, and only the first half (forgetting) of the update equation is implemented. This state of affairs continues until the strength has fallen below the lower limit, at which point the (re-) learning starts again-that is, they are incremented in the normal fashion. With the right parameter values, it is possible to get nice forced-choice probability correct values, even for the AN/BN null choice.

The reason one gets the right null choice is because there is considerable overlap of the strengths between the boundaries. There is also a question about underlying processes. To implement this algorithm, one would have to estimate not just the strength of an item, but also its first derivative (rate of change). Rate of change could be inferred from test-trial increments, but experimental data make this seem unlikely because of time constraints (Hintzman, 1994; Yonelinas, Hockley, \& Murdock, 1992).

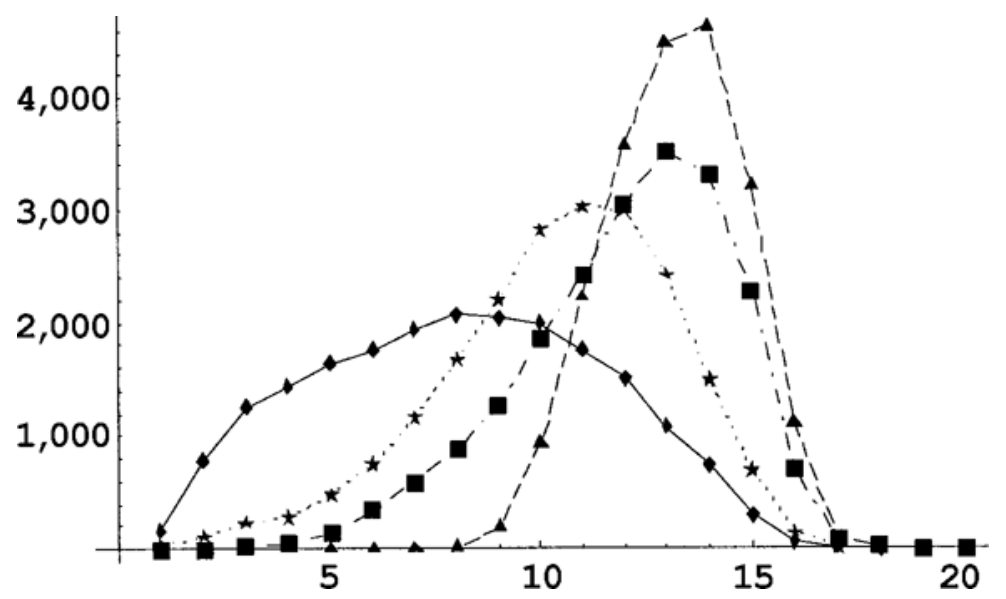

Fig A1. Strength distributions for AN, BN, BO, and AO for the first context update equation given above. 